\title{
On maxitive integration
}

\author{
Marco E. G. V. Cattaneo \\ Department of Mathematics, University of Hull \\ m. cattaneo@hull.ac.uk
}

\begin{abstract}
A functional is said to be maxitive if it commutes with the (pointwise) supremum operation. Such functionals find application in particular in decision theory and related fields. In the present paper, maxitive functionals are characterized as integrals with respect to maxitive measures (also known as possibility measures or idempotent measures). These maxitive integrals are then compared with the usual additive and nonadditive integrals on the basis of some important properties, such as convexity, subadditivity, and the law of iterated expectations.
\end{abstract}

Keywords: maxitive measures, nonadditive integrals, Choquet integral, convexity, subadditivity, law of iterated expectations.

\section{Introduction}

The standard integral of a function with respect to a probability measure (i.e., its expectation) can be interpreted as a weighted average of the function values. Such averages play a central role in the theory of decision making under risk, in which decisions are selected by maximizing expected utility or minimizing expected loss. However, in practice decisions are often selected on the basis of best-case or worst-case evaluations. Such evaluations are described by integrals that are maxitive (i.e., the integral of a pointwise maximum of functions is the maximum of their integrals), while the standard integral is additive (i.e., the integral of a pointwise sum of functions is the sum of their integrals). More generally, maxitive integrals appear as aggregation functionals in many fields of application, such as operations research, information fusion, or control theory (see for example Grabisch et al., 2009; Torra and Narukawa, 2007; Calvo et al., 2002).

Maxitive integrals can be seen as extensions of maxitive measures (i.e., the measure of a union of sets is the maximum of their measures). These nonadditive measures play a central role in possibility theory and tropical or idempotent mathematics (they are also called possibility measures or idempotent measures), but have been studied also in other contexts (see for instance Dubois and Prade, 1988; Kolokoltsov and Maslov, 1997; Wang and Klir, 2009). Contrary to the case of additivity, the countable (or even uncountable) maxitivity of measures and integrals does not pose particular difficulties, and measurability restrictions are thus unnecessary. This is discussed in the next section.

The rest of the paper can be divided into two parts. The first part (corresponding to Sections 3-5) studies the integral of nonnegative functions that 
was introduced by Shilkret (1971) and is characterized by maxitivity and positive homogeneity. Some of its properties, such as subadditivity and the law of iterated expectations, are derived and compared with corresponding properties of alternative definitions of integral. In particular, new results about the Choquet integral (Choquet, 1954; Denneberg, 1994) are also presented: necessary and sufficient conditions for countable comonotonic additivity and for the law of iterated expectations.

The second part corresponds to Section 6 and pursues the definition of a maxitive integral for all real functions. In fact, the Shilkret integral cannot be extended in a satisfactory way to a maxitive integral of real functions. Instead, the integral characterized by maxitivity and additive homogeneity is introduced and named convex integral. The reason for the name is its convexity, which is derived besides other properties, such as the law of iterated expectations. The convex integral is strictly related to the idempotent integral of tropical or idempotent mathematics (Kolokoltsov and Maslov, 1997) and to convex measures of risk (Föllmer and Schied, 2011).

\section{Maxitive measures}

Let $\Omega$ be a nonempty set, and let $\mathcal{A} \subseteq \mathcal{P}(\Omega)$ be a collection of subsets of $\Omega$. When $\kappa$ is a cardinal, $\mathcal{A}$ is said to be closed under $\kappa$-union if and only if $\bigcup \mathcal{B} \in \mathcal{A}$ for all nonempty $\mathcal{B} \subseteq \mathcal{A}$ such that $|\mathcal{B}| \leq \kappa$. An extended real-valued set function $\mu: \mathcal{A} \rightarrow \overline{\mathbb{R}}$ is said to be $\kappa$-maxitive if and only if $\mu(\cup \mathcal{B})=\sup _{A \in \mathcal{B}} \mu(A)$ for all nonempty $\mathcal{B} \subseteq \mathcal{A}$ such that $|\mathcal{B}| \leq \kappa$ and $\cup \mathcal{B} \in \mathcal{A}$. Furthermore, $\mu$ is said to be monotonic if and only if $\mu(A) \leq \mu(B)$ for all $A, B \in \mathcal{A}$ such that $A \subseteq B$. Monotonicity is implied by finite maxitivity (i.e., 2-maxitivity), while countable maxitivity (i.e., $\aleph_{0}$-maxitivity) was assumed by Shilkret (1971) in his definition of maxitive measures.

Theorem 1. Let $\kappa$ be a cardinal, and let $\mu: \mathcal{A} \rightarrow \overline{\mathbb{R}}$ be $\kappa$-maxitive. If $\mathcal{A}$ is closed under $\kappa$-union, or $\mathcal{A}$ is a ring (i.e., closed under finite union and finite intersection), then there is a $\kappa$-maxitive extension of $\mu$ to $\mathcal{P}(\Omega)$.

Proof. The cases with $\kappa \leq 1$ or $\mathcal{A}=\varnothing$ are trivial, so assume $\kappa>1$ and $\mathcal{A} \neq \varnothing$. Consider first the case with $\mathcal{A}$ closed under $\kappa$-union, and define $\mu^{\prime}$ : $A \mapsto \inf _{B \in \mathcal{A}: A \subseteq B} \mu(B)$ on $\mathcal{P}(\Omega)$, where inf $\varnothing=+\infty$. Since $\mu$ is monotonic, $\mu^{\prime}$ is a monotonic extension of $\mu$. Therefore, in order to prove the $\kappa$-maxitivity of $\mu^{\prime}$, it suffices to show $\mu^{\prime}(\bigcup \mathcal{B}) \leq \sup _{A \in \mathcal{B}} \mu^{\prime}(A)$ for all nonempty $\mathcal{B} \subseteq \mathcal{P}(\Omega)$ such that $|\mathcal{B}| \leq \kappa$ and $\sup _{A \in \mathcal{B}} \mu^{\prime}(A)<+\infty$. When $\mathcal{B}$ is such a set, for each $A \in \mathcal{B}$ and each $\varepsilon \in \mathbb{R}_{>0}$ there is an $A_{\varepsilon} \in \mathcal{A}$ such that $A \subseteq A_{\varepsilon}$ and $\mu\left(A_{\varepsilon}\right)<\mu^{\prime}(A)+\varepsilon$, and thus

$$
\mu^{\prime}(\bigcup \mathcal{B}) \leq \inf _{\varepsilon \in \mathbb{R}>0} \mu\left(\bigcup_{A \in \mathcal{B}} A_{\varepsilon}\right) \leq \inf _{\varepsilon \in \mathbb{R}_{>0}} \sup _{A \in \mathcal{B}}\left(\mu^{\prime}(A)+\varepsilon\right)=\sup _{A \in \mathcal{B}} \mu^{\prime}(A) .
$$

Now let $\mathcal{A}$ be a ring and let $\kappa$ be infinite (the case with finite $\kappa$ has already been considered above). If $\mathcal{B}, \mathcal{B}^{\prime} \subseteq \mathcal{A}$ are two nonempty sets with cardinality at most $\kappa$, and $\bigcup \mathcal{B}=\bigcup \mathcal{B}^{\prime}$, then

$$
\sup _{A \in \mathcal{B}} \mu(A)=\sup _{A \in \mathcal{B}} \mu\left(\bigcup_{B \in \mathcal{B}^{\prime}}(A \cap B)\right)=\sup _{A \in \mathcal{B}} \sup _{B \in \mathcal{B}^{\prime}} \mu(A \cap B)=\sup _{B \in \mathcal{B}^{\prime}} \mu(B) .
$$


Hence, the function $\tilde{\mu}: \bigcup \mathcal{B} \mapsto \sup _{A \in \mathcal{B}} \mu(A)$ on $\tilde{\mathcal{A}}=\{\bigcup \mathcal{B}: \mathcal{B} \subseteq \mathcal{A}, 0<|\mathcal{B}| \leq \kappa\}$ is a well-defined extension of $\mu$. In order to complete the proof of the theorem, it suffices to show that $\tilde{\mathcal{A}}$ is closed under $\kappa$-union and $\tilde{\mu}$ is $\kappa$-maxitive, and this follows easily from the fact that (assuming the axiom of choice) the product of two infinite cardinals is their maximum.

Since the focus of the present paper is on maxitive measures and integrals, Theorem 1 implies that measurability restrictions are unnecessary, and $\mathcal{A}=\mathcal{P}(\Omega)$ can always be assumed. Moreover, in order to simplify the following results, only nonnegative real-valued set functions $\mu: \mathcal{P}(\Omega) \rightarrow \mathbb{R}_{>0}$ are considered, and without real loss of generality $\mu(\Omega)=1$ is imposed. A set function $\mu: \mathcal{P}(\Omega) \rightarrow \overline{\mathbb{R}}$ is said to be a capacity on $\Omega$ (in the most general sense) if and only if $\mu$ is monotonic, $\mu(\varnothing)=0$, and $\mu(\Omega)=1$.

Capacities $\mu$ on $\Omega$ are often used in applications as quantitative descriptions of uncertain belief or information about $\omega \in \Omega$. The larger the value $\mu(A)$, the larger the plausibility of $\omega \in A$, or the larger the implausibility of $\omega \notin$ $A$. This is in agreement with the monotonicity of $\mu$, while the requirements $\mu(\varnothing)=0$ and $\mu(\Omega)=1$ can be interpreted as meaning that $\omega \in \varnothing$ is impossible and that nothing speaks against $\omega \in \Omega$, respectively. For example, finitely additive capacities are the quantitative descriptions of uncertain belief used in the Bayesian theory (de Finetti, 1974-1975; Savage, 1972), while countably additive capacities are used in standard probability theory (Kolmogorov, 1956).

As quantitative descriptions of uncertain belief or information, maxitive capacities play a central role in possibility theory (Zadeh, 1978; Dubois and Prade, 1988), but they also appear for instance as consonant plausibility functions in the theory of belief functions (Shafer, 1976), or as supremum preserving upper probabilities in the theory of imprecise probabilities (de Cooman and Aeyels, 1999). Moreover, the description of uncertain belief by means of maxitive capacities also corresponds for example to the descriptions by means of degrees of potential surprise (Shackle, 1949), or of degrees of support by eliminative induction (Cohen, 1966). Of particular importance in statistical applications is the fact that the likelihood (ratio) of composite hypotheses is a maxitive capacity (Neyman and Pearson, 1928; Cattaneo, 2013a).

A capacity $\mu$ on $\Omega$ has a particularly simple description when it is $\kappa$-maxitive with $\kappa$ the cardinality of $\Omega$. In fact, $\mu$ is then completely described by its values on the singletons: $\mu(A)=\sup _{\omega \in A} \mu(\{\omega\})$ for all nonempty $A \subseteq \Omega$. This implies in particular the $\kappa$-maxitivity of $\mu$ for all cardinals $\kappa$, also called complete maxitivity. For example, in statistics, the likelihood of composite hypotheses is a completely maxitive capacity: $\Lambda(\mathcal{H})=\sup _{\theta \in \mathcal{H}} L(\theta)$ for all composite hypotheses $\mathcal{H} \subseteq \Theta$, where $\Theta$ is a set of simple hypotheses, and $L$ is the (relative) likelihood function on $\Theta$.

\section{Characterization of integrals}

Let $\mathcal{F}$ be the set of all extended real-valued functions on $\Omega$, and let $\mathcal{F}^{+} \subset \mathcal{F}$ be the subset of all nonnegative functions. When $\mathcal{S} \subseteq \mathcal{F}$ is a set of functions and $\kappa$ is a cardinal, a functional $F: \mathcal{S} \rightarrow \overline{\mathbb{R}}$ is said to be $\kappa$-maxitive if and only if $F\left(\sup _{f \in \mathcal{T}} f\right)=\sup _{f \in \mathcal{T}} F(f)$ for all nonempty $\mathcal{T} \subseteq \mathcal{S}$ such that $|\mathcal{T}| \leq \kappa$ and $\sup _{f \in \mathcal{T}} f \in \mathcal{S}$, where $\sup _{f \in \mathcal{T}} f$ denotes the pointwise supremum of the functions 
in $\mathcal{T}$. Furthermore, $F$ is said to be monotonic if and only if $F(f) \leq F(g)$ for all $f, g \in \mathcal{S}$ such that $f \leq g$ holds pointwise. As in the case of measures, monotonicity is implied by finite maxitivity.

When functions $f \in \mathcal{S}$ are interpreted as descriptions of the (uncertain, since dependent on $\omega \in \Omega$ ) utility or loss of possible decisions, monotonic functionals $F: \mathcal{S} \rightarrow \overline{\mathbb{R}}$ can represent their evaluation on the basis of some criterion, and decision making then corresponds to maximizing or minimizing $F(f)$. For example, the Bayesian theory prescribes the use of the expectation functional (i.e., the integral with respect to the finitely additive capacity describing the uncertain belief about $\omega \in \Omega$ ), and decision making consists in maximizing the expected utility or minimizing the expected loss. This corresponds to evaluating decisions by averaging their possible consequences. By contrast, maxitive functionals correspond to best-case or worst-case evaluations of decisions. A simple example of completely maxitive functional on $\mathcal{F}$ is the supremum $f \mapsto \sup _{\omega \in \Omega} f(\omega)$, which corresponds to decision making by maximax utility or minimax loss. Weighted versions of these decision criteria correspond to the completely maxitive functionals $f \mapsto \sup _{\omega \in \Omega} f(\omega) w(\omega)$ on $\mathcal{F}^{+}$, where $w: \Omega \rightarrow \mathbb{R}_{>0}$ is a weighting function.

In order to simplify the interpretation, the consequences of decisions are expressed in terms of loss (or minus utility) in the rest of the paper. Hence, maxitive functionals represent worst-case evaluations of decisions. This can be seen as follows. If $F(f)$ is a worst-case evaluation of a function $f \in \mathcal{F}$, then for each nonempty $A \subset \Omega$, it is the maximum (i.e., the worst-case) of the evaluations $F_{A}(f)$ and $F_{\Omega \backslash A}(f)$ of $f$ on $A$ and $\Omega \backslash A$, respectively. Therefore, given two functions $f, g \in \mathcal{F}$ and a nonempty set $A \subset \Omega$ such that $f \geq g$ and $f \leq g$ hold pointwise on $A$ and $\Omega \backslash A$, respectively, since the evaluations are monotonic, the evaluation of the pointwise maximum $f \vee g$ of $f$ and $g$ satisfies

$$
\begin{aligned}
F(f \vee g) & =\max \left\{F_{A}(f), F_{\Omega \backslash A}(g)\right\}=\max \left\{F_{A}(f), F_{A}(g), F_{\Omega \backslash A}(g), F_{\Omega \backslash A}(f)\right\} \\
& =\max \{F(f), F(g)\} .
\end{aligned}
$$

Hence, worst-case evaluations of decisions are represented by finitely maxitive functionals (infinite maxitivity can be obtained analogously by considering infinite partitions of $\Omega$ ).

The Shilkret integral of a function $f \in \mathcal{F}^{+}$with respect to a capacity $\mu$ on $\Omega$ is denoted by $\int^{\mathrm{S}} f \mathrm{~d} \mu$ and is defined as

$$
\int^{\mathrm{S}} f \mathrm{~d} \mu=\sup _{x \in \mathbb{R}_{>0}} x \mu(\{\omega \in \Omega: f(\omega)>x\}) .
$$

The Shilkret integral has a particularly simple expression when $\mu$ is completely maxitive: $\int^{\mathrm{S}} f \mathrm{~d} \mu=\sup _{\omega \in \Omega: \mu(\{\omega\})>0} f(\omega) \mu(\{\omega\})$ for all $f \in \mathcal{F}^{+}$. As noted above, in statistics, the likelihood of composite hypotheses is a completely maxitive capacity $\Lambda$ on $\Theta$, and thus the Shilkret integral with respect to $\Lambda$ corresponds to decision making by likelihood-weighted minimax loss: $\int{ }^{\mathrm{S}} f \mathrm{~d} \Lambda=$ $\sup _{\theta \in \Theta} f(\theta) L(\theta)$, where $f: \Theta \rightarrow \mathbb{R}_{\geq 0}$ describes the loss of a possible decision and $L$ is the likelihood function on $\bar{\Theta}$ (Cattaneo, 2013a).

Example 1. Let $\mu$ be the capacity on $\Omega=\mathbb{R}$ such that $\mu(A)=1$ when $\sup A=$ $+\infty$, and $\mu(A)=1 / 2$ otherwise, for all nonempty sets $A \subseteq \mathbb{R}$. Then $\mu$ is finitely 
maxitive, but not countably maxitive, and for all $f \in \mathcal{F}^{+}$,

$$
\int^{\mathrm{S}} f \mathrm{~d} \mu=\max \left\{\limsup _{x \rightarrow+\infty} f(x), \frac{1}{2} \sup _{x \in \mathbb{R}} f(x)\right\} .
$$

The function in $\mathcal{F}$ taking the same values as $f \in \mathcal{F}$ on $A \subseteq \Omega$ and the value $x$ on $\Omega \backslash A$ is denoted by $A_{f, x}$. In particular, $A_{1,0}$ is the usual indicator function of $A$. Since $\int{ }^{\mathrm{S}} A_{1,0} \mathrm{~d} \mu=\mu(A)$ for all $A \subseteq \Omega$, the Shilkret integral can be seen as an extension of the capacity $\mu$ (see also Cattaneo, 2014). The next lemma (which is a direct consequence of the definitions) shows that this extension maintains maxitivity.

Lemma 1. Let $\mu$ be a capacity on $\Omega$, and let $\kappa$ be a cardinal. The functional $f \mapsto \int^{\mathrm{S}} f \mathrm{~d} \mu$ on $\mathcal{F}^{+}$is $\kappa$-maxitive if and only if $\mu$ is $\kappa$-maxitive.

It is interesting to compare the properties of the Shilkret integral with the properties of alternative definitions of integral. In particular, an important integral with respect to nonadditive measures is the Choquet integral (see for example Choquet, 1954; Denneberg, 1994). The Choquet integral of a function $f \in \mathcal{F}^{+}$with respect to a capacity $\mu$ on $\Omega$ is denoted by $\int{ }^{\mathrm{C}} f \mathrm{~d} \mu$ and is defined as

$$
\int^{\mathrm{C}} f \mathrm{~d} \mu=\int_{0}^{+\infty} \mu(\{\omega \in \Omega: f(\omega)>x\}) \mathrm{d} x,
$$

where the integral on the right-hand side is a well-defined improper Riemann integral.

Example 2. Let $\mu$ be the capacity on $\Omega=\mathbb{R}$ defined in Example 1. Then, for all $f \in \mathcal{F}^{+}$,

$$
\int^{\mathrm{C}} f \mathrm{~d} \mu=\frac{1}{2}\left(\limsup _{x \rightarrow+\infty} f(x)\right)+\frac{1}{2}\left(\sup _{x \in \mathbb{R}} f(x)\right) .
$$

The standard integral with respect to additive measures can also be generalized as follows to the case of nonadditive measures. The standard integral of a function $f \in \mathcal{F}^{+}$with respect to a capacity $\mu$ on $\Omega$ is denoted by $\int f \mathrm{~d} \mu$ and is defined as

$$
\int f \mathrm{~d} \mu=\sum_{x \in f[\Omega]} x \mu(\{\omega \in \Omega: f(\omega)=x\})
$$

when $f$ is a simple function (i.e., its image $f[\Omega]$ is a finite subset of $\mathbb{R}$ ), while otherwise it is defined as

$$
\int f \mathrm{~d} \mu=\sup _{s \in \mathcal{S}_{f}} \int s \mathrm{~d} \mu
$$

where $\mathcal{S}_{f}$ denotes the set of all simple functions $s \in \mathcal{F}^{+}$such that $s \leq f$ holds pointwise. This corresponds to the usual integral when $\mu$ is finitely additive (see for example Bhaskara Rao and Bhaskara Rao, 1983), and to the Lebesgue integral when $\mu$ is countably additive (see for instance Ash, 1972).

Example 3. Let $\mu$ be the capacity on $\Omega=\mathbb{R}$ defined in Example 1. Then, in particular, for all non-simple $f \in \mathcal{F}^{+}$,

$$
\int f \mathrm{~d} \mu=\frac{1}{2} \sum_{x \in \mathbb{R}} f(x) .
$$


Let $\mathcal{U} \subset \mathcal{F}$ be the subset of all functions bounded above, and define $\mathcal{U}^{+}=$ $\mathcal{U} \cap \mathcal{F}^{+}$. The next theorem characterizes the above three integrals as functionals on the set $\mathcal{U}^{+}$of all bounded, nonnegative real-valued functions on $\Omega$. The following definitions are needed for this characterization. Two functions $f, g \in \mathcal{F}$ are said to be comonotonic if and only if $f(\omega)>f\left(\omega^{\prime}\right)$ implies $g(\omega) \geq g\left(\omega^{\prime}\right)$ for all $\omega, \omega^{\prime} \in \Omega$. When $\mathcal{S} \subseteq \mathcal{F}$ is a set of functions, a functional $F: \mathcal{S} \rightarrow \overline{\mathbb{R}}$ is said to be finitely (comonotonic) additive if and only if $F(f+g)=F(f)+F(g)$ for all (comonotonic) $f, g \in \mathcal{S}$ such that $f+g$ and $F(f)+F(g)$ are well-defined and $f+g \in \mathcal{S}$. Furthermore, $F$ is said to be positively homogeneous if and only if $F(\alpha f)=\alpha F(f)$ for all $\alpha \in \mathbb{R}_{>0}$ and all $f \in \mathcal{S}$ such that $\alpha f \in \mathcal{S}$.

Theorem 2. Let $F: \mathcal{U}^{+} \rightarrow \overline{\mathbb{R}}_{\geq 0}$ be a functional such that $F(1)=1$, and define $\mu: A \mapsto F\left(A_{1,0}\right)$ on $\mathcal{P}(\Omega)$.

(i) $F$ is finitely additive if and only if $\mu$ is a finitely additive capacity and $F: f \mapsto \int f \mathrm{~d} \mu$.

(ii) $F$ is finitely comonotonic additive and monotonic if and only if $\mu$ is a capacity and $F: f \mapsto \int^{\mathrm{C}} f \mathrm{~d} \mu$.

(iii) $F$ is finitely maxitive and positively homogeneous if and only if $\mu$ is a finitely maxitive capacity and $F: f \mapsto \int^{\mathrm{s}} f \mathrm{~d} \mu$.

Proof. See Bhaskara Rao and Bhaskara Rao (1983, Chapter 4) for the "if" part of (i). The "only if" part can be proved as follows. Since $F$ is finitely additive, it is also monotonic, and $\mu$ is a finitely additive capacity. The finite additivity of $F$ implies also $F(\alpha f)=\alpha F(f)$ for all $\alpha \in \mathbb{Q}_{>0}$ and all $f \in \mathcal{U}^{+}$. The positive homogeneity of $F$ follows then from its monotonicity. Therefore, $F(f)=\int f \mathrm{~d} \mu$ holds for all simple functions $f \in \mathcal{U}^{+}$. The result for all functions $f \in \mathcal{U}^{+}$ follows from the monotonicity of $F$ and the fact that for each $\varepsilon \in \mathbb{R}_{>0}$ there is a simple function $s \in \mathcal{U}^{+}$such that $s \leq f \leq s+\varepsilon$ holds pointwise.

The "if" part of (ii) is proved in Schmeidler (1986, Remark 4). For the "only if" part, since $F$ is finitely comonotonic additive and monotonic, $\mu$ is a capacity, and the rest can be shown as in Schmeidler (1986, Proof of the Theorem).

The "if" part of (iii) is a direct consequence of the definitions (and Lemma 1). The "only if" part can be proved as follows. Since $F$ is finitely maxitive and positively homogeneous, it is also monotonic, and $\mu$ is a finitely maxitive capacity. Moreover, $F(f)=\max _{x \in f[\Omega]} x \mu(\{\omega \in \Omega: f(\omega)=x\})=\int{ }^{\mathrm{s}} f \mathrm{~d} \mu$ holds for all simple functions $f \in \mathcal{U}^{+}$. The result for all functions $f \in \mathcal{U}^{+}$follows as in the proof of (i).

For a (monotonic) functional describing the evaluation of the loss or utility of possible decisions, positive homogeneity means that the unit in which these are measured is of no concern in the decision making. In all three cases of Theorem 2, the functional $F$ is monotonic and positively homogeneous. Hence, the characterizing properties of the standard, Choquet, and Shilkret integrals on bounded, nonnegative functions are finite additivity, finite comonotonic additivity, and finite maxitivity, respectively. These properties do not characterize the integrals on unbounded, nonnegative functions, but their countable versions do, as shown in the next theorem. The following definitions are needed for this characterization. When $\mathcal{S} \subseteq \mathcal{F}$ is a set of functions, a functional $F: \mathcal{S} \rightarrow \overline{\mathbb{R}}$ is said to be countably (comonotonic) additive if and only if $F\left(\sum_{n \in \mathbb{N}} f_{n}\right)=\sum_{n \in \mathbb{N}} F\left(f_{n}\right)$ 
for all sequences $\left(f_{n}\right)_{n \in \mathbb{N}}$ of (pairwise comonotonic) functions $f_{n} \in \mathcal{S}$ such that $\sum_{n \in \mathbb{N}} f_{n}$ and $\sum_{n \in \mathbb{N}} F\left(f_{n}\right)$ are well-defined and $\sum_{n \in \mathbb{N}} f_{n} \in \mathcal{S}$. A capacity $\mu$ on $\Omega$ is said to be 0 -continuous if and only if $\mu\left(\bigcup_{n \in \mathbb{N}} A_{n}\right)=0$ for all nondecreasing sequences $\left(A_{n}\right)_{n \in \mathbb{N}}$ of sets $A_{n} \subset \Omega$ with $\mu\left(A_{n}\right)=0$.

Theorem 3. Let $F: \mathcal{F}^{+} \rightarrow \overline{\mathbb{R}}_{\geq 0}$ be a functional such that $F(1)=1$, and define $\mu: A \mapsto F\left(A_{1,0}\right)$ on $\mathcal{P}(\Omega)$.

(i) $F$ is countably additive if and only if $\mu$ is a countably additive capacity and $F: f \mapsto \int f \mathrm{~d} \mu$.

(ii) $F$ is countably comonotonic additive and monotonic if and only if $\mu$ is a 0-continuous capacity and $F: f \mapsto \int^{\mathrm{C}} f \mathrm{~d} \mu$.

(iii) $F$ is countably maxitive and positively homogeneous if and only if $\mu$ is a countably maxitive capacity and $F: f \mapsto \int \mathrm{S} f \mathrm{~d} \mu$.

Proof. See Ash (1972, Corollary 1.6.4) for the "if" part of (i). The "only if" part can be proved as follows. Since $F$ is countably additive, it is also monotonic and finitely additive, and $\mu$ is a countably additive capacity. Thus, Theorem 2 (i) implies $F(f)=\int f \mathrm{~d} \mu$ for all $f \in \mathcal{U}^{+}$. The result for all $f \in \mathcal{F}^{+}$follows from the fact that there is a sequence $\left(f_{n}\right)_{n \in \mathbb{N}}$ of functions $f_{n} \in \mathcal{U}^{+}$such that $f=\sum_{n \in \mathbb{N}} f_{n}$, and therefore $F$ is uniquely determined by $\mu$.

The "if" part of (ii) can be proved as follows. The monotonicity of $F$ is a direct consequence of the definitions. In order to show that $F$ is also countably comonotonic additive, let $\left(f_{n}\right)_{n \in \mathbb{N}}$ be a sequence of pairwise comonotonic functions $f_{n} \in \mathcal{F}^{+}$, and define $f=\sum_{n \in \mathbb{N}} f_{n}$. Then $\int^{\mathrm{C}} f \mathrm{~d} \mu \geq \sum_{n \in \mathbb{N}} \int^{\mathrm{C}} f_{n} \mathrm{~d} \mu$ follows from the finite comonotonic additivity of $F$, which is proved for example in Wakker (1989, Theorem 2) and Cattaneo (2007, Theorem 2.25). Assume thus $\sum_{n \in \mathbb{N}} \int^{\mathrm{C}} f_{n} \mathrm{~d} \mu<+\infty$, and define the set $I=\{\omega \in \Omega: f(\omega)=+\infty\}$, the values $x_{n}=\sup _{\omega \in \Omega \backslash I} f_{n}(\omega)$, and the functions $g_{n}=f_{n} \wedge x_{n}$ and $g=\sum_{n \in \mathbb{N}} g_{n}$ on $\Omega$, where $\wedge$ denotes the pointwise minimum.

If $\mu(I)=0$, then $\int^{\mathrm{C}} f_{n} \mathrm{~d} \mu=\int{ }^{\mathrm{C}} g_{n} \mathrm{~d} \mu$ for all $n \in \mathbb{N}$, and $\int^{\mathrm{C}} f \mathrm{~d} \mu=\int{ }^{\mathrm{C}} g \mathrm{~d} \mu$, because the functions $f_{n}$ are pairwise comonotonic, and therefore $f_{n}(\omega) \geq x_{n}$ and $g_{n}(\omega)=x_{n}$ for all $n \in \mathbb{N}$ and all $\omega \in I$. Hence, in order to prove the "if" part of (ii), it suffices to show $\mu(I)=0$ and $\int{ }^{\mathrm{C}} g \mathrm{~d} \mu=\sum_{n \in \mathbb{N}} \int{ }^{\mathrm{C}} g_{n} \mathrm{~d} \mu$.

In order to show $\mu(I)=0$ when $I \neq \varnothing$, define for each $\omega \in I$ the set $I_{\omega}=\bigcap_{n \in \mathbb{N}}\left\{\omega^{\prime} \in I: f_{n}\left(\omega^{\prime}\right) \geq f_{n}(\omega)\right\}$. Then $\mu\left(I_{\omega}\right)=0$ for all $\omega \in I$, because $\sum_{n \in \mathbb{N}} \int{ }^{\mathrm{C}}\left(I_{\omega}\right)_{f_{n}(\omega), 0} \mathrm{~d} \mu \leq \sum_{n \in \mathbb{N}} \int^{\mathrm{C}} f_{n} \mathrm{~d} \mu<+\infty$. Furthermore, since the functions $f_{n}$ are pairwise comonotonic, the sets $I_{\omega}$ are nested (i.e., $I_{\omega} \subseteq I_{\omega^{\prime}}$ or $I_{\omega^{\prime}} \subseteq I_{\omega}$ for all $\left.\omega, \omega^{\prime} \in I\right)$, and $\left\{\omega^{\prime} \in I: f_{n}\left(\omega^{\prime}\right)>f_{n}(\omega)\right\} \subseteq I_{\omega}$ for all $n \in \mathbb{N}$ and all $\omega \in I$. Hence, if there is an $n \in \mathbb{N}$ such that the function $f_{n}$ does not take the value $\inf _{\omega \in I} f_{n}(\omega)$ in $I$, then $I=\bigcup_{n \in \mathbb{N}} I_{\omega_{n}}$ for a sequence $\left(\omega_{n}\right)_{n \in \mathbb{N}}$ of points $\omega_{n} \in I$ such that $\lim _{n \rightarrow \infty} f_{n}\left(\omega_{n}\right)=\inf _{\omega \in I} f_{n}(\omega)$. Therefore, in this case $\mu(I)=0$ because $\mu$ is 0 -continuous and the sets $I_{\omega}$ are nested. On the other hand, if for each $n \in \mathbb{N}$ there is a point $\omega_{n} \in I$ such that $f_{n}\left(\omega_{n}\right)=\min _{\omega \in I} f_{n}(\omega)$, then either $I=\bigcup_{n \in \mathbb{N}} I_{\omega_{n}}$, or there is a point $\omega \in I$ such that $f_{n}(\omega)=\min _{\omega^{\prime} \in I} f_{n}\left(\omega^{\prime}\right)$ for all $n \in \mathbb{N}$, and thus $I=I_{\omega}$. In both cases, $\mu(I)=0$.

In order to show $\int{ }^{\mathrm{C}} g \mathrm{~d} \mu=\sum_{n \in \mathbb{N}} \int{ }^{\mathrm{C}} g_{n} \mathrm{~d} \mu$, it suffices to prove that there is a sequence $\left(u_{n}\right)_{n \in \mathbb{N}}$ of continuous, nondecreasing functions $u_{n}: \overline{\mathbb{R}}_{\geq 0} \rightarrow \overline{\mathbb{R}}_{\geq 0}$ 
with $g_{n}=u_{n} \circ g$ and such that $\sum_{n \in \mathbb{N}} u_{n}$ is the identity function on $\overline{\mathbb{R}}_{\geq 0}$. The desired result then follows from Denneberg (1994, Proposition 4.1) and Ash (1972, Corollary 1.6.4). The existence of the functions $u_{n}$ can be proved analogously to Denneberg (1994, Proposition 4.5), since the functions $g_{n}$ are pairwise comonotonic, and $g(\omega)=+\infty$ is possible only if $g_{n}(\omega)=x_{n}$ for all $n \in \mathbb{N}$. This concludes the proof of the "if" part of (ii).

The "only if" part of (ii) can be proved as follows. Since $F$ is countably comonotonic additive and monotonic, it is also finitely comonotonic additive, and $\mu$ is a capacity. In order to show that $\mu$ is 0 -continuous, let $\left(A_{n}\right)_{n \in \mathbb{N}}$ be an nondecreasing sequence of sets $A_{n} \subset \Omega$ with $\mu\left(A_{n}\right)=0$. Then $\mu\left(\bigcup_{n \in \mathbb{N}} A_{n}\right) \leq$ $\sum_{n \in \mathbb{N}} \mu\left(A_{n}\right)=0$, because the functions $\left(A_{n}\right)_{1,0}$ are pairwise comonotonic. Furthermore, Theorem 2 (ii) implies $F(f)=\int^{\mathrm{C}} f \mathrm{~d} \mu$ for all $f \in \mathcal{U}^{+}$. The result for all $f \in \mathcal{F}^{+}$follows from the fact that there is a sequence $\left(f_{n}\right)_{n \in \mathbb{N}}$ of pairwise comonotonic functions $f_{n} \in \mathcal{U}^{+}$such that $f=\sum_{n \in \mathbb{N}} f_{n}$, and therefore $F$ is uniquely determined by $\mu$.

The "if" part of (iii) is a direct consequence of the definitions (and Lemma 1). The "only if" part follows from Theorem 2 (iii) and the fact that for each $f \in \mathcal{F}^{+}$ there is a sequence $\left(f_{n}\right)_{n \in \mathbb{N}}$ of functions $f_{n} \in \mathcal{U}^{+}$such that $f=\sup _{n \in \mathbb{N}} f_{n}$, and thus $F$ is uniquely determined by $\mu$.

The necessary and sufficient condition of 0-continuity of the capacity for the countable comonotonic additivity of the Choquet integral seems to be a new result, though related results were obtained by Wu and Zhou (2006). An alternative characterization of the Shilkret integral on unbounded, nonnegative functions, in which countable maxitivity is weakened to countable comonotonic maxitivity was given by Benvenuti et al. (2002, see also Benvenuti and Vivona, 2000). In this characterization, the Choquet and Shilkret integrals appear as special cases of a more general notion of integral, but the stronger condition of continuity from below of the capacity is assumed instead of its 0 -continuity.

The next corollary shows that the Choquet integral is a generalization to the case of nonadditive measures of the standard integral with respect to additive measures, while the Shilkret integral is an alternative definition of integral also in the case of additive measures. A set function $\mu: \mathcal{P}(\Omega) \rightarrow \overline{\mathbb{R}}$ is said to be binary if and only if it takes only two values. Hence, in particular, a capacity is binary if and only if it takes only the values 0 and 1 .

Corollary 1. Let $\mu$ be a capacity on $\Omega$.

(i) $\int f \mathrm{~d} \mu=\int^{\mathrm{C}} f \mathrm{~d} \mu$ for all $f \in \mathcal{F}^{+}$if and only if $\mu$ is finitely additive.

(ii) $\int{ }^{\mathrm{C}} f \mathrm{~d} \mu=\int{ }^{\mathrm{S}} f \mathrm{~d} \mu$ for all $f \in \mathcal{F}^{+}$if and only if $\mu$ is binary.

(iii) $\int{ }^{\mathrm{S}} f \mathrm{~d} \mu=\int f \mathrm{~d} \mu$ for all $f \in \mathcal{F}^{+}$if and only if $\mu$ is finitely additive and binary.

Proof. The "if" part of (i) can be proved as follows. Theorem 2 (i) implies that the functional $f \mapsto \int f \mathrm{~d} \mu$ on $\mathcal{U}^{+}$is finitely additive, and thus also monotonic. Hence, Theorem 2 (ii) implies $\int f \mathrm{~d} \mu=\int{ }^{\mathrm{C}} f \mathrm{~d} \mu$ for all $f \in \mathcal{U}^{+}$. The result for all $f \in \mathcal{F}^{+}$follows from the fact that for both definitions of integral, the integral of $f$ is the limit of the one of $f \wedge n$ as $n \in \mathbb{N}$ tends to infinity. The "if" part of (ii) is a direct consequence of the definitions, while the "if" part of (iii) is implied by the corresponding parts of (i) and (ii). 
In order to prove the "only if" parts, let $A, B \subseteq \Omega$ be disjoint and $x \in \mathbb{R}_{>0}$, and define $f_{x}=A_{x+1,0}+B_{1,0}$. Then $\int f_{x} \mathrm{~d} \mu=\mu(B)+(x+1) \mu(A)$, while $\int{ }^{\mathrm{C}} f_{x} \mathrm{~d} \mu=\mu(A \cup B)+x \mu(A)$ and $\int{ }^{\mathrm{S}} f_{x} \mathrm{~d} \mu=\max \{\mu(A \cup B),(x+1) \mu(A)\}$. The limits of these integrals as $x$ tends to 0 imply the finite additivity of $\mu$ in (i) and (iii). The binarity of $\mu$ in (ii) and (iii) follows from the fact that when $B=\Omega \backslash A$, the function $x \mapsto \int^{\mathrm{S}} f_{x} \mathrm{~d} \mu$ is affine on $\mathbb{R}_{>0}$ if and only if $\mu(A) \in\{0,1\}$.

\section{Subadditivity}

A particularly important result for nonadditive integrals is the characterization of the nonadditive measures with respect to which they are at least subadditive (see for example Denneberg, 1994, Chapter 6). When $\mathcal{S} \subseteq \mathcal{F}$ is a set of functions, a functional $F: \mathcal{S} \rightarrow \overline{\mathbb{R}}$ is said to be finitely subadditive if and only if $F(f+g) \leq$ $F(f)+F(g)$ for all $f, g \in \mathcal{S}$ such that $f+g$ and $F(f)+F(g)$ are well-defined and $f+g \in \mathcal{S}$. Furthermore, $F$ is said to be finitely convex if and only if $F(\lambda f+(1-\lambda) g) \leq \lambda F(f)+(1-\lambda) F(g)$ for all $\lambda \in(0,1)$ and all $f, g \in \mathcal{S}$ such that $f+g$ and $F(f)+F(g)$ are well-defined and $\lambda f+(1-\lambda) g \in \mathcal{S}$. A set function $\mu: \mathcal{P}(\Omega) \rightarrow \mathbb{R}$ is said to be submodular if and only if $\mu(A \cup B)+\mu(A \cap B) \leq$ $\mu(A)+\mu(B)$ for all $A, B \subseteq \Omega$. Hence, in particular, all finitely additive capacities and all finitely maxitive capacities are submodular.

Theorem 4. Let $\mu$ be a capacity on $\Omega$.

(i) The functional $f \mapsto \int f \mathrm{~d} \mu$ on $\mathcal{F}^{+}$is finitely subadditive if and only if $\mu$ is finitely additive.

(ii) The functional $f \mapsto \int{ }^{\mathrm{C}} f \mathrm{~d} \mu$ on $\mathcal{F}^{+}$is finitely subadditive if and only if $\mu$ is submodular.

(iii) The functional $f \mapsto \int^{\mathrm{S}} f \mathrm{~d} \mu$ on $\mathcal{F}^{+}$is finitely subadditive if and only if $\mu$ is finitely maxitive.

Proof. In order to prove the "if" part of (i), it suffices to show that if $f, g \in \mathcal{F}^{+}$ and $s \in \mathcal{S}_{f+g}$, then $\int s \mathrm{~d} \mu \leq \int f \mathrm{~d} \mu+\int g \mathrm{~d} \mu$. This inequality is implied by Theorem 2 (i), since $s \wedge f$ and $s-s \wedge f$ are bounded, and $s \wedge f \leq f$ and $s-s \wedge f \leq g$ hold pointwise. The "only if" part of (i) follows from the fact that for all disjoint $A, B \subseteq \Omega$,

$$
\begin{aligned}
2 \mu(A)+\mu(B)=\int\left((A \cup B)_{1,0}+A_{1,0}\right) \mathrm{d} \mu \leq \mu(A \cup B)+\mu(A) \\
=\int\left(A_{1,0}+B_{1,0}\right) \mathrm{d} \mu+\mu(A) \leq 2 \mu(A)+\mu(B) .
\end{aligned}
$$

See Denneberg (1994, Chapter 6) for the proof of (ii).

In order to prove the "if" part of (iii), it suffices to show that if $f, g \in \mathcal{F}^{+}$and $x \in \mathbb{R}_{>0}$, then $x \mu(\{\omega \in \Omega: f(\omega)+g(\omega)>x\}) \leq \int{ }^{\mathrm{S}} f \mathrm{~d} \mu+\int{ }^{\mathrm{S}} g \mathrm{~d} \mu$. Following Shilkret (1971, page 113), assume thus $\int^{\mathrm{S}} f \mathrm{~d} \mu+\int{ }^{\mathrm{S}} g \mathrm{~d} \mu<+\infty$, and note that 
for all $\lambda \in(0,1)$,

$$
\begin{aligned}
x \mu & (\{\omega \in \Omega: f(\omega)+g(\omega)>x\}) \\
& \leq x \mu(\{\omega \in \Omega: f(\omega)>\lambda x\} \cup\{\omega \in \Omega: g(\omega)>(1-\lambda) x\}) \\
& =\max \left\{x \mu\left(\left\{\omega \in \Omega: \frac{1}{\lambda} f(\omega)>x\right\}\right), x \mu\left(\left\{\omega \in \Omega: \frac{1}{1-\lambda} g(\omega)>x\right\}\right)\right\} \\
& \leq \max \left\{\frac{1}{\lambda} \int^{\mathrm{S}} f \mathrm{~d} \mu, \frac{1}{1-\lambda} \int^{\mathrm{S}} g \mathrm{~d} \mu\right\} .
\end{aligned}
$$

The desired result is obtained by letting $\lambda$ tend to 0 or 1 when $\int^{\mathrm{s}} f \mathrm{~d} \mu=0$ or $\int^{\mathrm{S}} g \mathrm{~d} \mu=0$, respectively, and by setting

$$
\lambda=\frac{\int^{\mathrm{s}} f \mathrm{~d} \mu}{\int \mathrm{S}^{\mathrm{S}} \mathrm{d} \mu+\int^{\mathrm{S}} g \mathrm{~d} \mu}
$$

otherwise. The "only if" part of (iii) follows from the fact that for all disjoint $A, B \subseteq \Omega$ and all $\lambda \in(0,1)$, if $\lambda \mu(A \cup B)>\max \{\mu(A), \mu(B)\}$, then

$$
\begin{aligned}
\mu(A \cup B) \leq \int^{\mathrm{S}}\left(A_{1,0}+B_{\lambda, 0}\right) \mathrm{d} \mu & +\int^{\mathrm{S}} B_{1-\lambda, 0} \mathrm{~d} \mu \\
& =\lambda \mu(A \cup B)+(1-\lambda) \mu(B)<\mu(A \cup B) .
\end{aligned}
$$

Convexity is a very important property for functionals $F: \mathcal{S} \rightarrow \overline{\mathbb{R}}$ describing the evaluation of the loss (or minus utility) $f \in \mathcal{S}$ of possible (investment) decisions. In fact, the convexity of the evaluation functional then represents the desirability of investment diversification (see for example Föllmer and Schied, 2011; Artzner et al., 1999). For positively homogeneous functionals on $\mathcal{F}^{+}$or $\mathcal{F}$, convexity and subadditivity are equivalent.

Theorem 4 gives necessary and sufficient conditions on the capacities for the finite subadditivity (or convexity) of the integrals. Countable subadditivity (or convexity) is implied by finite subadditivity (or convexity) when the integrals satisfy a monotone convergence theorem. When $\mathcal{S} \subseteq \mathcal{F}$ is a set of functions, a functional $F: \mathcal{S} \rightarrow \overline{\mathbb{R}}$ is said to be countably subadditive if and only if $F\left(\sum_{n \in \mathbb{N}} f_{n}\right) \leq \sum_{n \in \mathbb{N}} F\left(f_{n}\right)$ for all sequences $\left(f_{n}\right)_{n \in \mathbb{N}}$ of functions $f_{n} \in \mathcal{S}$ such that $\sum_{n \in \mathbb{N}} f_{n}$ and $\sum_{n \in \mathbb{N}} F\left(f_{n}\right)$ are well-defined and $\sum_{n \in \mathbb{N}} f_{n} \in \mathcal{S}$. The functional $F$ is said to be countably convex if and only if $F\left(\sum_{n \in \mathbb{N}} \lambda_{n} f_{n}\right) \leq \sum_{n \in \mathbb{N}} \lambda_{n} F\left(f_{n}\right)$ for all sequences $\left(\lambda_{n}\right)_{n \in \mathbb{N}}$ of values $\lambda_{n} \in(0,1)$ such that $\sum_{n \in \mathbb{N}} \lambda_{n}=1$ and all sequences $\left(f_{n}\right)_{n \in \mathbb{N}}$ of functions $f_{n} \in \mathcal{S}$ such that $\sum_{n \in \mathbb{N}} \lambda_{n} f_{n}$ and $\sum_{n \in \mathbb{N}} \lambda_{n} F\left(f_{n}\right)$ are well-defined and $\sum_{n \in \mathbb{N}} \lambda_{n} f_{n} \in \mathcal{S}$. Furthermore, $F$ is said to satisfy monotone convergence if and only if it is monotonic and $F\left(\lim _{n \rightarrow \infty} f_{n}\right)=\lim _{n \rightarrow \infty} F\left(f_{n}\right)$ for all pointwise nondecreasing sequences $\left(f_{n}\right)_{n \in \mathbb{N}}$ of functions $f_{n} \in \mathcal{S}$ such that $\lim _{n \rightarrow \infty} f_{n} \in \mathcal{S}$, where $\lim _{n \rightarrow \infty} f_{n}$ denotes the pointwise limit of the sequence. A monotonic set function $\mu: \mathcal{P}(\Omega) \rightarrow \overline{\mathbb{R}}$ is said to be continuous from below if and only if $\mu\left(\bigcup_{n \in \mathbb{N}} A_{n}\right)=\lim _{n \rightarrow \infty} \mu\left(A_{n}\right)$ for all nondecreasing sequences $\left(A_{n}\right)_{n \in \mathbb{N}}$ of sets $A_{n} \subset \Omega$.

Lemma 2. Let $\mu$ be a capacity on $\Omega$. 
(i) The functional $f \mapsto \int f \mathrm{~d} \mu$ on $\mathcal{F}^{+}$satisfies monotone convergence if and only if $\mu$ is countably additive.

(ii) The functional $f \mapsto \int{ }^{\mathrm{C}} f \mathrm{~d} \mu$ on $\mathcal{F}^{+}$satisfies monotone convergence if and only if $\mu$ is continuous from below.

(iii) The functional $f \mapsto \int{ }^{\mathrm{S}} f \mathrm{~d} \mu$ on $\mathcal{F}^{+}$satisfies monotone convergence if and only if $\mu$ is continuous from below.

Proof. The "if" part of (i) follows from Theorem 3 (i). See Denneberg (1994, Theorem 8.1) for the "if" part of (ii). The "if" part of (iii) can be proved analogously: for all pointwise nondecreasing sequences $\left(f_{n}\right)_{n \in \mathbb{N}}$ of functions $f_{n} \in \mathcal{F}^{+}$,

$$
\begin{aligned}
\int^{\mathrm{S}}\left(\lim _{n \rightarrow \infty} f_{n}\right) \mathrm{d} \mu & =\sup _{x \in \mathbb{R}_{>0}} x \mu\left(\bigcup_{n \in \mathbb{N}}\left\{\omega \in \Omega: f_{n}(\omega)>x\right\}\right) \\
& =\sup _{x \in \mathbb{R}_{>0}} x \sup _{n \in \mathbb{N}} \mu\left(\left\{\omega \in \Omega: f_{n}(\omega)>x\right\}\right)=\lim _{n \rightarrow \infty} \int^{\mathrm{S}} f_{n} \mathrm{~d} \mu .
\end{aligned}
$$

For the "only if" parts, the continuity from below of $\mu$ is a direct consequence of the fact that all three integrals are extensions of $\mu$ (when sets are identified with their indicator functions). In (i) $\mu$ is also finitely additive (and thus countably additive), since $\mu(A \cup B)=\lim _{\lambda \uparrow 1} \int\left(A_{\lambda, 0}+B_{1,0}\right) \mathrm{d} \mu$ for all disjoint $A, B \subseteq \Omega$.

Corollary 2. Let $\mu$ be a capacity on $\Omega$.

(i) The functional $f \mapsto \int f \mathrm{~d} \mu$ on $\mathcal{F}^{+}$is countably subadditive if and only if $\mu$ is countably additive.

(ii) The functional $f \mapsto \int{ }^{\mathrm{C}} f \mathrm{~d} \mu$ on $\mathcal{F}^{+}$is countably subadditive if $\mu$ is submodular and continuous from below.

(iii) The functional $f \mapsto \int{ }^{\mathrm{S}} f \mathrm{~d} \mu$ on $\mathcal{F}^{+}$is countably subadditive if $\mu$ is countably maxitive.

Corollary 2 gives sufficient conditions on the capacities for the countable subadditivity (or convexity) of the integrals. For the Choquet and Shilkret integrals these conditions are not necessary, as shown in the next example. Necessary and sufficient conditions on the capacities for the countable subadditivity (or convexity) of the Choquet and Shilkret integrals are an open problem.

Example 4. The capacity $\mu$ on $\Omega=\mathbb{R}$ defined in Example 1 is finitely maxitive, but not countably maxitive (and thus submodular, but not continuous from below). However, both functionals $f \mapsto \int{ }^{\mathrm{C}} f \mathrm{~d} \mu$ and $f \mapsto \iint^{\mathrm{S}} f \mathrm{~d} \mu$ on $\mathcal{F}^{+}$are countably subadditive. In order to prove this, it suffices to show

$$
\limsup _{x \rightarrow+\infty} \sum_{n \in \mathbb{N}} f_{n}(x) \leq \sum_{n \in \mathbb{N}} \limsup _{x \rightarrow+\infty} f_{n}(x)
$$

for all sequences $\left(f_{n}\right)_{n \in \mathbb{N}}$ of functions $f_{n} \in \mathcal{F}^{+}$such that $\sum_{n \in \mathbb{N}} \sup _{x \in \mathbb{R}} f_{n}(x)<$ $+\infty$. This property follows from the fact that for each $\varepsilon \in \mathbb{R}_{>0}$ there is a finite $\mathcal{N} \subset \mathbb{N}$ such that $\sum_{n \in \mathbb{N} \backslash \mathcal{N}} \sup _{x \in \mathbb{R}} f_{n}(x) \leq \varepsilon$, and therefore

$$
\limsup _{x \rightarrow+\infty} \sum_{n \in \mathbb{N}} f_{n}(x) \leq \limsup _{x \rightarrow+\infty}\left(\varepsilon+\sum_{n \in \mathcal{N}} f_{n}(x)\right) \leq \varepsilon+\sum_{n \in \mathcal{N}} \limsup _{x \rightarrow+\infty} f_{n}(x) .
$$




\section{Law of iterated expectations}

For an integral describing the evaluation (or expectation) of a function with respect to a measure, it is important to satisfy a law of iterated expectations (or evaluations). In fact, backward induction can then be applied and conditional evaluations can be used as an intermediate step toward the (unconditional) evaluation of a function. When $F: \mathcal{F}^{+} \rightarrow \overline{\mathbb{R}}_{\geq 0}$ is a functional such that $\mu: A \mapsto F\left(A_{1,0}\right)$ on $\mathcal{P}(\Omega)$ is a capacity, the conditional evaluation by $F$ of a function $f \in \mathcal{F}^{+}$with respect to a partition $\mathcal{C} \subseteq \mathcal{P}(\Omega)$ of $\Omega$ is denoted by $F(f \mid \mathcal{C})$ and is defined as $F(f \mid \mathcal{C})=\sum_{C \in \mathcal{C}} C_{F(f \mid C), 0}$, where $F(f \mid C)=0$ when $\mu(C)=0$, and

$$
F(f \mid C)=\frac{F\left(C_{f, 0}\right)}{\mu(C)}
$$

otherwise. When $\kappa$ is a cardinal, $F$ is said to be $\kappa$-decomposable if and only if $F(F(f \mid \mathcal{C}))=F(f)$ for all $f \in \mathcal{F}^{+}$and all partitions $\mathcal{C} \subseteq \mathcal{P}(\Omega)$ of $\Omega$ such that $|\mathcal{C}| \leq \kappa$.

Lemma 3. Let $\mu$ be a capacity on $\Omega$, and let $\kappa$ be a cardinal. The functional $f \mapsto \int^{\mathrm{S}} f \mathrm{~d} \mu$ on $\mathcal{F}^{+}$is $\kappa$-decomposable if $\mu$ is $\kappa$-maxitive.

Proof. Lemma 1 implies that the functional $F: f \mapsto \int^{\mathrm{S}} f \mathrm{~d} \mu$ on $\mathcal{F}^{+}$is $\kappa$ maxitive. Let $f \in \mathcal{F}^{+}$be a function, and let $\mathcal{C} \subseteq \mathcal{P}(\Omega)$ be a partition of $\Omega$ such that $|\mathcal{C}| \leq \kappa$. Then $F(f \mid C) \mu(C)=F\left(C_{f, 0}\right)$ for all $C \in \mathcal{C}$, and therefore

$$
\int^{\mathrm{S}}\left(\int^{\mathrm{S}} f \mathrm{~d} \mu \mid \mathcal{C}\right) \mathrm{d} \mu=\sup _{C \in \mathcal{C}} \int^{\mathrm{S}} C_{F(f \mid C), 0} \mathrm{~d} \mu=\sup _{C \in \mathcal{C}} \int^{\mathrm{S}} C_{f, 0} \mathrm{~d} \mu=\int^{\mathrm{S}} f \mathrm{~d} \mu .
$$

The next theorem gives necessary and sufficient conditions on the capacities for the finite decomposability (i.e., 2-decomposability) of the integrals. It generalizes in particular a result of Yoo (1991, see also Dominiak, 2013), by showing that the Choquet integral can satisfy a (finite) law of iterated expectations only if it corresponds to the standard integral (when the capacity is finitely additive) or to the Shilkret integral (when the capacity is finitely maxitive and binary).

Theorem 5. Let $\mu$ be a capacity on $\Omega$ with positive values for at least 3 pairwise disjoint subsets of $\Omega$.

(i) The functional $f \mapsto \int f \mathrm{~d} \mu$ on $\mathcal{F}^{+}$is finitely decomposable if and only if $\mu$ is finitely additive.

(ii) The functional $f \mapsto \int^{\mathrm{C}} f \mathrm{~d} \mu$ on $\mathcal{F}^{+}$is finitely decomposable if and only if $\mu$ is finitely additive or $\mu$ is finitely maxitive and binary.

(iii) The functional $f \mapsto \int^{\mathrm{S}} f \mathrm{~d} \mu$ on $\mathcal{F}^{+}$is finitely decomposable if and only if $\mu$ is finitely maxitive.

Proof. The "if" part of (i) can be proved analogously to Lemma 3, using the finite additivity instead of the $\kappa$-maxitivity. The finite additivity of $f \mapsto \int f \mathrm{~d} \mu$ on $\mathcal{F}^{+}$follows from Theorem 4 (i) and the fact that for all $f, g \in \mathcal{F}^{+}$, if $s \in \mathcal{S}_{f}$ and $s^{\prime} \in \mathcal{S}_{g}$, then $s+s^{\prime} \in \mathcal{S}_{f+g}$, and thus $\int s \mathrm{~d} \mu+\int s^{\prime} \mathrm{d} \mu \leq \int(f+g) \mathrm{d} \mu$ is implied by Theorem 2 (i). The "if" part of (iii) is a special case of Lemma 3, 
while the "if" part of (ii) is implied by the corresponding parts of (i) and (iii) using Corollary 1 (i) and (ii), respectively.

For the "only if" parts, first note that for all $A, B \in \Omega$, if $\mu(B)=0$, then $\mu(A \cup B)=\mu(A)$, since the conditional evaluations of $(A \cup B)_{1,0}$ with respect to the partition $\{A, \Omega \backslash A\}$ take the value 0 on $\Omega \backslash A$. In particular, if $\mu$ is binary, then it is finitely maxitive. Furthermore, for all $A \subseteq \Omega$, at least one of $A$ and $\Omega \backslash A$ is the union of two disjoint subsets with positive capacity values, because $\mu$ has positive values for at least 3 pairwise disjoint subsets of $\Omega$, and the previous result implies that for each of these 3 subsets, the intersections with $A$ and $\Omega \backslash A$ cannot both have capacity value 0 .

The "only if" part of (i) can be proved by contradiction as follows. Assume that there are $A, B \subseteq \Omega$ disjoint and such that $\mu(A \cup B) \neq \mu(A)+\mu(B)$. Then $\mu(A)>0$ and $\mu(B)>0$, and thus

$$
\int\left(\int(A \cup B)_{1,0} \mathrm{~d} \mu \mid\{A, \Omega \backslash A\}\right) \mathrm{d} \mu=\int\left(A_{1,0}+\frac{\mu(B)}{\mu(\Omega \backslash A)}(\Omega \backslash A)_{1,0}\right) \mathrm{d} \mu
$$

implies $\mu(B)=\mu(\Omega \backslash A)$ and $\mu(A \cup B)=1$. Hence, $\mu(A)+\mu(\Omega \backslash A)<1$, and at least one of $A$ and $\Omega \backslash A$, say $A$, is the union of two disjoint subsets with positive capacity values: $C$ and $A \backslash C$. Therefore,

$$
\int\left(\int\left(\frac{\mu(A)}{\mu(C)} C_{1,0}+(\Omega \backslash A)_{1,0}\right) \mathrm{d} \mu \mid\{A, \Omega \backslash A\}\right) \mathrm{d} \mu=\int 1 \mathrm{~d} \mu
$$

implies $\mu(A)=\mu(C)$, and $\mu(A)=\mu(A \backslash C)$ follows by symmetry. As shown above, $\mu(A) \neq \mu(A)+\mu(A \backslash C)$ implies $\mu(A)=1$, leading to a contradiction:

$$
\begin{aligned}
1+2 \mu(\Omega \backslash A) & =\int\left(\int A_{1,2} \mathrm{~d} \mu \mid\{C, \Omega \backslash C\}\right) \mathrm{d} \mu=\int C_{1,1+2 \mu(\Omega \backslash A)} \mathrm{d} \mu \\
& =2+2 \mu(\Omega \backslash A) .
\end{aligned}
$$

In order to prove the "only if" part of (ii), it suffices to show that if $\mu$ is not finitely additive, then it is binary. Assume thus that $\mu$ is not finitely additive: there are $A, B \subseteq \Omega$ disjoint and such that $\mu(A \cup B) \neq \mu(A)+\mu(B)$. Then $\mu(A)>0$ and $\mu(B)>0$, and for all $x \in \mathbb{R}_{>0}$,

$$
\begin{aligned}
\mu(A \cup B)+x \mu(B) & =\int^{\mathrm{C}}\left(\int^{\mathrm{C}}\left((A \cup B)_{1,0}+B_{x, 0}\right) \mathrm{d} \mu \mid\{A, \Omega \backslash A\}\right) \mathrm{d} \mu \\
& =\int^{\mathrm{C}}\left(A_{1,0}+\frac{(1+x) \mu(B)}{\mu(\Omega \backslash A)}(\Omega \backslash A)_{1,0}\right) \mathrm{d} \mu .
\end{aligned}
$$

This implies $\mu(B)=\mu(\Omega \backslash A)$ and thus $\mu(A \cup B)=1$, because $\mu(B)<\mu(\Omega \backslash A)$ would imply

$$
\mu(A \cup B)+x \mu(B)=\mu(A)+\frac{(1+x) \mu(B)}{\mu(\Omega \backslash A)}(1-\mu(A))
$$

for sufficiently small $x \in \mathbb{R}_{>0}$, which means $\mu(\Omega \backslash A)=1-\mu(A)$ and $\mu(A \cup B)=$ $\mu(A)+\mu(B)$. Hence, $\mu(A)+\mu(\Omega \backslash A) \neq 1$. Now, for all $C \subseteq \Omega \backslash A$, if $\mu(C)>0$, 
then

$$
\begin{aligned}
\mu(A \cup C) & +\mu(\Omega \backslash A)-\mu(C) \\
= & \int^{\mathrm{C}}\left(\int^{\mathrm{C}}\left(A_{1,0}+\frac{\mu(\Omega \backslash A)}{\mu(C)} C_{1,0}\right) \mathrm{d} \mu \mid\{A, \Omega \backslash A\}\right) \mathrm{d} \mu=\int^{\mathrm{C}} 1 \mathrm{~d} \mu
\end{aligned}
$$

implies $\mu(A \cup C) \neq \mu(A)+\mu(C)$, and as shown above, from this follows $\mu(C)=$ $\mu(\Omega \backslash A)$. By symmetry, for all $C \subseteq A$, if $\mu(C)>0$, then $\mu(C)=\mu(A)$. In order to complete the proof of the "only if" part of (ii), it suffices to show $\mu(A)=\mu(\Omega \backslash A)=1$, because $\mu(D) \in\{0,1\}$ for all $D \subseteq \Omega$ then follows from $\mu(D \cap A), \mu(D \backslash A) \in\{0,1\}$. In order to show $\mu(A)=\mu(\Omega \backslash A)=1$, remember that at least one of $A$ and $\Omega \backslash A$, say $A$, is the union of two disjoint subsets with positive capacity values: $E$ and $A \backslash E$. Hence, $\mu(A)=\mu(E)=\mu(A \backslash E)$, and as shown above, $\mu(A) \neq \mu(E)+\mu(A \backslash E)$ implies $\mu(A)=1$. Finally, $\mu(\Omega \backslash A)=1$ follows from

$$
\begin{aligned}
1+\mu(\Omega \backslash A) & =\int^{\mathrm{C}}\left(\int^{\mathrm{C}}\left(E_{1,0}+(\Omega \backslash A)_{2,0}\right) \mathrm{d} \mu \mid\{E, \Omega \backslash E\}\right) \mathrm{d} \mu \\
& =\int^{\mathrm{C}} E_{1,2 \mu(\Omega \backslash A)} \mathrm{d} \mu=\max \{1,2 \mu(\Omega \backslash A)\} .
\end{aligned}
$$

The "only if" part of (iii) can be proved by contradiction as follows. Assume that there are $A, B \subseteq \Omega$ disjoint and such that $\mu(A \cup B)>\max \{\mu(A), \mu(B)\}$. Then $\mu(A)>0$ and $\mu(B)>0$, and thus

$$
\begin{aligned}
\int^{\mathrm{S}}\left(\int^{\mathrm{S}}(A \cup B)_{1,0} \mathrm{~d} \mu \mid\{A, \Omega \backslash A\}\right) \mathrm{d} \mu \\
\quad=\int^{\mathrm{S}}\left(A_{1,0}+\frac{\mu(B)}{\mu(\Omega \backslash A)}(\Omega \backslash A)_{1,0}\right) \mathrm{d} \mu=\max \left\{\frac{\mu(B)}{\mu(\Omega \backslash A)}, \mu(A)\right\}
\end{aligned}
$$

implies $\mu(\Omega \backslash A)<1$ and $\mu(B)=\mu(\Omega \backslash A) \mu(A \cup B)$. Therefore,

$$
\begin{aligned}
& \max \{\mu(A \cup B), \mu(\Omega \backslash A)\} \\
&=\int^{\mathrm{S}}\left(\int^{\mathrm{S}}\left(A_{1,0}+\frac{\mu(\Omega \backslash A)}{\mu(B)} B_{1,0}\right) \mathrm{d} \mu \mid\{A, \Omega \backslash A\}\right) \mathrm{d} \mu=\int^{\mathrm{S}} 1 \mathrm{~d} \mu
\end{aligned}
$$

implies $\mu(A \cup B)=1$ and $\mu(B)=\mu(\Omega \backslash A)$. Hence, $\max \{\mu(A), \mu(\Omega \backslash A)\}<1$, and at least one of $A$ and $\Omega \backslash A$, say $A$, is the union of two disjoint subsets with positive capacity values: $C$ and $A \backslash C$, with $\mu(\Omega \backslash C) \geq \mu(\Omega \backslash(A \backslash C))$. As shown above, if $\mu((\Omega \backslash A) \cup C)>\max \{\mu(\Omega \backslash A), \mu(C)\}$, then $\mu(\Omega \backslash A)=$ $\mu(\Omega \backslash C)$, and thus $\mu(\Omega \backslash(A \backslash C))>\mu(\Omega \backslash C)$. Therefore, $\mu((\Omega \backslash A) \cup C)=$ $\max \{\mu(\Omega \backslash A), \mu(C)\}$, but this leads to a contradiction:

$$
\begin{aligned}
1 & =\int^{\mathrm{S}}\left(\int^{\mathrm{S}}\left((A \backslash C)_{1,0}+\frac{1}{\mu(C)} C_{1,0}+\frac{1}{\mu(\Omega \backslash A)}(\Omega \backslash A)_{1,0}\right) \mathrm{d} \mu \mid\{A, \Omega \backslash A\}\right) \\
& =\int^{\mathrm{S}}\left(\frac{1}{\mu(A)} A_{1,0}+\frac{1}{\mu(\Omega \backslash A)}(\Omega \backslash A)_{1,0}\right) \mathrm{d} \mu>1 .
\end{aligned}
$$


Countable decomposability (i.e., $\aleph_{0}$-decomposability) is implied by finite decomposability when the integrals satisfy monotone convergence.

Corollary 3. Let $\mu$ be a capacity on $\Omega$ with positive values for at least 3 pairwise disjoint subsets of $\Omega$.

(i) The functional $f \mapsto \int f \mathrm{~d} \mu$ on $\mathcal{F}^{+}$is countably decomposable if and only if $\mu$ is countably additive.

(ii) The functional $f \mapsto \iint^{\mathrm{C}} f \mathrm{~d} \mu$ on $\mathcal{F}^{+}$is countably decomposable if and only if $\mu$ is countably additive or $\mu$ is countably maxitive and binary.

(iii) The functional $f \mapsto \int{ }^{\mathrm{S}} f \mathrm{~d} \mu$ on $\mathcal{F}^{+}$is countably decomposable if $\mu$ is countably maxitive.

Proof. The "if" parts can be proved as follows using Lemma 2 and Theorem 5. Let $F: \mathcal{F}^{+} \rightarrow \overline{\mathbb{R}}_{>0}$ be a finitely decomposable functional satisfying monotone convergence, let $f \in \mathcal{F}^{+}$be a function, and let $\left(\mathcal{C}_{n}\right)_{n \in \mathbb{N}}$ be a nondecreasing sequence of finite sets $\mathcal{C}_{n} \subseteq \mathcal{P}(\Omega)$ such that $\mathcal{C}=\bigcup_{n \in \mathbb{N}} \mathcal{C}_{n}$ is a partition of $\Omega$. Then

$$
\begin{aligned}
F(F(f \mid \mathcal{C})) & =\lim _{n \rightarrow \infty} F\left(\sum_{C \in \mathcal{C}_{n}} C_{F(f \mid C), 0}\right) \\
& =\lim _{n \rightarrow \infty} F\left(F\left(\left(\bigcup \mathcal{C}_{n}\right)_{f, 0} \mid \mathcal{C}_{n} \cup\left\{\Omega \backslash \bigcup \mathcal{C}_{n}\right\}\right)\right) \\
& =F\left(\lim _{n \rightarrow \infty}\left(\bigcup \mathcal{C}_{n}\right)_{f, 0}\right)=F(f) .
\end{aligned}
$$

The "only if" part of (i) can be proved by contradiction as follows. Assume that there is a sequence $\left(A_{n}\right)_{n \in \mathbb{N}}$ of pairwise disjoint sets $A_{n} \subseteq \Omega$ such that $\mu\left(\bigcup_{n \in \mathbb{N}} A_{n}\right) \neq \sum_{n \in \mathbb{N}} \mu\left(A_{n}\right)$. Theorem 5 (i) implies that $\mu$ is finitely additive, and thus $\mu\left(\bigcup_{n \in \mathbb{N}} A_{n}\right)>\sum_{n \in \mathbb{N}} \mu\left(A_{n}\right)$. Therefore, infinitely many $A_{n}$ have positive capacity values, since

$$
\int\left(\int\left(\bigcup_{n \in \mathbb{N}} A_{n}\right)_{1,0} \mathrm{~d} \mu \mid\left\{A_{n}: n \in \mathbb{N}\right\}\right) \mathrm{d} \mu=\int\left(\bigcup_{n \in \mathbb{N}: \mu\left(A_{n}\right)>0} A_{n}\right)_{1,0} \mathrm{~d} \mu .
$$

Hence, $\mu$ takes infinitely many values, and Schervish et al. (1984, Theorem 3.1) implies that there are a set $A \subseteq \Omega$ and a countable partition $\mathcal{C}$ of $\Omega$ such that $\mu(A)>\sup _{C \in \mathcal{C}}\left(\int A_{1,0} \mathrm{~d} \mu \mid C\right)$, leading to a contradiction:

$$
\mu(A)=\int\left(\int A_{1,0} \mathrm{~d} \mu \mid \mathcal{C}\right) \mathrm{d} \mu \leq \int\left(\sup _{C \in \mathcal{C}}\left(\int A_{1,0} \mathrm{~d} \mu \mid C\right)\right) \mathrm{d} \mu<\mu(A) .
$$

For the "only if" part of (ii), Theorem 5 (ii) implies that $\mu$ is finitely additive or binary. If $\mu$ is finitely additive, then (i) and Corollary 1 (i) imply that $\mu$ is also countably additive. If $\mu$ is binary, then it is also countably maxitive when $\mu\left(\bigcup_{n \in \mathbb{N}} A_{n}\right)=0$ for all sequences $\left(A_{n}\right)_{n \in \mathbb{N}}$ of pairwise disjoint sets $A_{n} \subset \Omega$ with $\mu\left(A_{n}\right)=0$, and this follows from

$$
\int^{\mathrm{C}}\left(\int^{\mathrm{C}}\left(\bigcup_{n \in \mathbb{N}} A_{n}\right)_{1,0} \mathrm{~d} \mu \mid\left\{A_{n}: n \in \mathbb{N}\right\}\right) \mathrm{d} \mu=\int^{\mathrm{C}} 0 \mathrm{~d} \mu .
$$


Corollary 3 gives sufficient conditions on the capacities for the countable decomposability of the integrals. For the Shilkret integral the condition may not be necessary, but the running example is not a counterexample.

Example 5. The capacity $\mu$ on $\Omega=\mathbb{R}$ defined in Example 1 is neither countably additive, nor countably maxitive, and the standard, Choquet, and Shilkret integrals with respect to $\mu$ are not countably decomposable. More generally, if a functional $F: \mathcal{F}^{+} \rightarrow \overline{\mathbb{R}}_{>0}$ is positively homogeneous and satisfies $F\left(A_{1,0}\right)=\mu(A)$ for all $A \subseteq \mathbb{R}$, then it is not countably decomposable. In order to prove this, define for each $n \in \mathbb{N}$ the set $C_{n}=\left\{n+e^{m}-e: m \in \mathbb{N}\right\}$. Since e is transcendental, the sets $C_{n}$ are pairwise disjoint, and thus

$$
\begin{aligned}
F\left(F\left(\mathbb{N}_{1,0} \mid\left\{C_{n}: n \in \mathbb{N}\right\} \cup\left\{\mathbb{R} \backslash \bigcup_{n \in \mathbb{N}} C_{n}\right\}\right)\right) & =F\left(\frac{1}{2}\left(\bigcup_{n \in \mathbb{N}} C_{n}\right)\right. \\
& =\frac{1}{2} \neq 1=F\left(\mathbb{N}_{1,0}\right) .
\end{aligned}
$$

\section{Maxitive integrals of real functions}

Theorem 2 (iii) characterizes the Shilkret integral as finitely maxitive, positively homogeneous functional on all bounded, nonnegative functions. But the next example shows that finite maxitivity and positive homogeneity are not sufficient to characterize an integral on all bounded functions.

Example 6. Let $\Omega=\mathbb{N}$, and let $F, F^{\prime}: \mathcal{F} \rightarrow \overline{\mathbb{R}}$ be the functionals defined by $F(f)=f(1)$ when $\sup _{n \in \mathbb{N}} f(n)<0$, and $F(f)=\max \{f(1), 0\}$ otherwise, and by $F^{\prime}(f)=f(1)$ when $f(n)<0$ for all $n \in \mathbb{N}$, and $F^{\prime}(f)=\max \{f(1), 0\}$ otherwise, respectively, for all $f \in \mathcal{F}$. Then $F, F^{\prime}$ are finitely maxitive and positively homogeneous, and $F(f)=F^{\prime}(f)$ for all simple functions $f \in \mathcal{F}$, with in particular $F(x)=F^{\prime}(x)=x$ for all $x \in \mathbb{R}$. However, $F \neq F^{\prime}$, since for instance $F(f)=0 \neq-1=F^{\prime}(f)$ when $f: n \mapsto-1 / n$.

The next theorem shows that not even countably maxitive, positively homogeneous functionals $F: \mathcal{F} \rightarrow \overline{\mathbb{R}}$ with $F( \pm 1)= \pm 1$ are uniquely determined by the capacity $\mu: A \mapsto F\left(A_{1,0}\right)$ on $\mathcal{P}(\Omega)$. A set function $\nu: \mathcal{P}(\Omega) \rightarrow \overline{\mathbb{R}}$ is said to be a penalty on $\Omega$ if and only if $\nu$ is monotonic, $\nu(\varnothing)=-\infty$, and $\nu(\Omega)=0$.

Theorem 6. Let $F: \mathcal{F} \rightarrow \overline{\mathbb{R}}$ be a functional such that $F(1)=1$ and $F(-1)=$ -1 , and define $\mu: A \mapsto F\left(A_{1,0}\right)$ and $\nu: A \mapsto F\left(A_{-1,-\infty}\right)+1$ on $\mathcal{P}(\Omega)$, and $\mathcal{D}=\left\{D \subset \Omega: F\left(D_{1,-\infty}\right)=-\infty\right\}$. Then $F$ is countably maxitive and positively homogeneous if and only if $\mu$ is a countably maxitive capacity, $\nu$ is a countably maxitive penalty, $\mathcal{D}$ is a $\sigma$-ideal such that $\mu(D)=0$ and $\nu(D)=-\infty$ for all $D \in \mathcal{D}$, and

$F: f \mapsto \begin{cases}\sup _{x \in \mathbb{R}_{>0}} x \mu(\{\omega \in \Omega: f(\omega)>x\}) & \text { if }\{\omega \in \Omega: f(\omega)>0\} \notin \mathcal{D}, \\ \sup _{x \in \mathbb{R}_{<0}} x(1-\nu(\{\omega \in \Omega: f(\omega)>x\})) & \text { if }\{\omega \in \Omega: f(\omega)>0\} \in \mathcal{D} .\end{cases}$

Proof. The "if" part can be proved as follows. Let $\mathcal{T} \subseteq \mathcal{F}$ be a set of functions such that $0<|\mathcal{T}| \leq \aleph_{0}$. Since $\mathcal{D}$ is a $\sigma$-ideal, $\left\{\omega \in \Omega: \sup _{f \in \mathcal{T}} f(\omega)>0\right\} \in \mathcal{D}$ if and only if $\{\omega \in \Omega: f(\omega)>0\} \in \mathcal{D}$ for all $f \in \mathcal{T}$. The countable maxitivity 
of $F$ follows now from the countable maxitivity of $\mu$ and $\nu$, while the positive homogeneity of $F$ is a direct consequence of the definitions.

For the "only if" part, since $F$ is countably maxitive and positively homogeneous, it is also monotonic, $\mu$ is a countably maxitive capacity, $\nu$ is a countably maxitive penalty, and $\mathcal{D}$ is a $\sigma$-ideal. Furthermore, if $D \in \mathcal{D}$, then $\mu(D)=F\left(D_{1,-\infty} \vee 0\right)=0$ and $\nu(D) \leq F\left(D_{1,-\infty}\right)+1=-\infty$. If $A \notin \mathcal{D}$, then $F\left(A_{1,-\infty}\right)=\mu(A)$, because $F\left(A_{1,-\infty}\right) \in \mathbb{R}_{<0}$ would imply $F\left(A_{2,-\infty}\right)=$ $2 F\left(A_{1,-\infty}\right)<F\left(A_{1,-\infty}\right)$. The desired result follows from the fact that for all $f \in \mathcal{F}$,

$$
\begin{aligned}
F(f) & =\sup _{x \in \mathbb{Q} \neq 0} F\left(\{\omega \in \Omega: f(\omega)>x\}_{x,-\infty}\right) \\
& =\max \left\{\begin{array}{l}
\sup _{x \in \mathbb{Q}_{>0}} x F\left(\{\omega \in \Omega: f(\omega)>x\}_{1,-\infty}\right), \\
\sup _{x \in \mathbb{Q}<0}(-x) F\left(\{\omega \in \Omega: f(\omega)>x\}_{-1,-\infty}\right)
\end{array}\right\},
\end{aligned}
$$

and thus $F$ is uniquely determined by $\mathcal{D}, \mu, \nu$.

The functionals $F: \mathcal{F} \rightarrow \overline{\mathbb{R}}$ considered in Theorem 6 extend the Shilkret integral to functions taking also negative values, since $F(f)=\int{ }^{\mathrm{s}} f \mathrm{~d} \mu$ for all $f \in \mathcal{F}^{+}$. Some non-maxitive extensions of the Shilkret integral to functions taking also negative values were introduced by Greco and Rindone (2013). The next lemma shows that all such extensions can be finitely subadditive only when $\mu$ is binary.

Lemma 4. Let $\mu$ be a capacity on $\Omega$, and let $F: \mathcal{F} \rightarrow \overline{\mathbb{R}}$ be a functional such that $F(-1)=-1$ and $F(f)=\int^{\mathrm{S}} f \mathrm{~d} \mu$ for all $f \in \mathcal{F}^{+}$. If $F$ is finitely subadditive, then $\mu$ is binary.

Proof. If $\mu$ is not binary, then there are an $A \subset \Omega$ with $\mu(A) \notin\{0,1\}$ and an $x \in \mathbb{R}_{>0}$ with $(1+x) \mu(A)<1$, and thus $F$ is not subadditive, because $F\left(A_{x, 0}\right)=x \mu(A)>1-1=F\left(A_{x+1,1}\right)+F(-1)$.

In order to make the functionals $F: \mathcal{F} \rightarrow \overline{\mathbb{R}}$ considered in Theorem 6 uniquely determined by the capacity $\mu: A \mapsto F\left(A_{1,0}\right)$ on $\mathcal{P}(\Omega)$, their homogeneity can be assumed instead of positive homogeneity. This means additionally assuming $F(-f)=-F(f)$ for all $f \in \mathcal{F}$. But the next lemma shows that this is only possible when $\mu$ is binary. When $\mathcal{S} \subseteq \mathcal{F}$ is a set of functions, a functional $F: \mathcal{S} \rightarrow \overline{\mathbb{R}}$ is said to be homogeneous if and only if $F(\alpha f)=\alpha F(f)$ for all $\alpha \in \mathbb{R}_{\neq 0}$ and all $f \in \mathcal{S}$ such that $\alpha f \in \mathcal{S}$.

Lemma 5. Let $F: \mathcal{F} \rightarrow \overline{\mathbb{R}}$ be a functional such that $F(1)=1$, and define $\mu: A \mapsto F\left(A_{1,0}\right)$ on $\mathcal{P}(\Omega)$. If $F$ is finitely maxitive and homogeneous, then $\mu$ is a finitely additive, binary capacity.

Proof. Since $F$ is finitely maxitive and homogeneous, it is also monotonic, and $\mu$ is a finitely maxitive capacity. The desired result follows from the fact that for all $A \subseteq \Omega$, not only $\max \{\mu(A), \mu(\Omega \backslash A)\}=1$, but also

$$
\min \{\mu(A), \mu(\Omega \backslash A)\}=-\max \left\{F\left(-A_{1,0}\right), F\left(-(\Omega \backslash A)_{1,0}\right)\right\}=0 .
$$

Corollary 4. Let $\Omega$ be a nonempty set such that there is no $\sigma$-complete, nonprincipal ultrafilter on $\Omega$, and let $F: \mathcal{F} \rightarrow \overline{\mathbb{R}}$ be a functional such that $F(1)=1$. Then $F$ is countably maxitive and homogeneous if and only if there is a point $\omega \in \Omega$ such that $F: f \mapsto f(\omega)$. 
Proof. The "if" part is a direct consequence of the definitions. The "only if" part can be proved as follows. Theorem 6 and Lemma 5 imply that the set function $\mu: A \mapsto F\left(A_{1,0}\right)$ on $\mathcal{P}(\Omega)$ is a countably additive, binary capacity, $\mathcal{D}=\left\{D \subset \Omega: F\left(D_{1,-\infty}\right)=-\infty\right\} \subseteq\{D \subset \Omega: \mu(D)=0\}$, and $F(f) \geq 0$ for all $f \in \mathcal{F}^{+}$. Thus $F(f)=\int^{\mathrm{s}} f \mathrm{~d} \mu$ for all $f \in \mathcal{F}^{+}$follows from Theorem 3 (iii). In particular, $F\left(A_{-1,-\infty}\right)=-\int^{\mathrm{S}} A_{1,+\infty} \mathrm{d} \mu=-1$ when $\mu(A)=1$, and $F\left(A_{-1,-\infty}\right)=-\infty$ otherwise, for all $A \subseteq \Omega$. Furthermore, if $D \subset \Omega$ with $\mu(D)=0$, then $D \in \mathcal{D}$, because $\min \left\{F\left(D_{1,-\infty}\right), 0\right\}=-\int \mathrm{S} D_{0,+\infty} \mathrm{d} \mu=-\infty$. Hence, the set $\mathcal{P}(\Omega) \backslash \mathcal{D}=\{A \subseteq \Omega: \mu(A)=1\}$ is a $\sigma$-complete ultrafilter on $\Omega$, and thus there is a point $\omega \in \Omega$ such that $\mathcal{P}(\Omega) \backslash \mathcal{D}=\{A \subseteq \Omega: \omega \in A\}$, and $F: f \mapsto f(\omega)$ follows then from Theorem 6 .

Theorem 6 implies that positive homogeneity is too weak a property to determine a maxitive integral of real functions with respect to a capacity, while Lemma 5 shows that homogeneity is too strong. Alternative strengthenings of positive homogeneity are possible, but rather arbitrary. It is more interesting to replace positive homogeneity by additive homogeneity. When $\mathcal{S} \subseteq \mathcal{F}$ is a set of functions, a functional $F: \mathcal{S} \rightarrow \overline{\mathbb{R}}$ is said to be additively homogeneous if and only if $F(f+\alpha)=F(f)+\alpha$ for all $\alpha \in \mathbb{R}$ and all $f \in \mathcal{S}$ such that $f+\alpha \in \mathcal{S}$. For a functional describing the evaluation of the loss or utility of possible decisions, additive homogeneity means that these can be measured on an interval scale, since the location of the zero point is of no concern in the decision making (Stevens, 1946).

The convex integral of a function $f \in \mathcal{F}$ with respect to a penalty $\nu$ on $\Omega$ is denoted by $\int^{\mathrm{X}} f \mathrm{~d} \nu$ and is defined as

$$
\int^{\mathrm{X}} f \mathrm{~d} \nu=\sup _{x \in \mathbb{R}}(x+\nu(\{\omega \in \Omega: f(\omega)>x\})) .
$$

The convex integral has a particularly simple expression when $\nu$ is completely maxitive: $\int^{\mathrm{X}} f \mathrm{~d} \nu=\sup _{\omega \in \Omega: \nu(\{\omega\})>-\infty}(f(\omega)+\nu(\{\omega\}))$ for all $f \in \mathcal{F}$. For example, in statistics, the log-likelihood of composite hypotheses is a completely maxitive penalty: $\ell(\mathcal{H})=\sup _{\theta \in \mathcal{H}} \ln L(\theta)$ for all composite hypotheses $\mathcal{H} \subseteq \Theta$, where $\ln 0=-\infty$ and, as above, $\Theta$ is a set of simple hypotheses and $L$ is the (relative) likelihood function on $\Theta$. Hence, the convex integral with respect to $\ell$ corresponds to decision making by log-likelihood-penalized minimax loss (or maximin utility): $\int^{\mathrm{x}} f \mathrm{~d} \ell=\sup _{\theta \in \Theta}(f(\theta)+\ln L(\theta))$, where $f: \Theta \rightarrow \mathbb{R}$ describes the loss (or minus utility) of a possible decision. Furthermore, the convex integral with respect to completely maxitive penalties is strictly related to the idempotent integral of tropical or idempotent mathematics (Kolokoltsov and Maslov, 1997) and to convex measures of risk (Föllmer and Schied, 2011). Note however that the notion of convex integral considered in the present paper (and in Cattaneo, 2014, 2013b) is different from the one introduced by Mesiar et al. (2015).

Example 7. Let $\nu$ be the penalty on $\Omega=\mathbb{R}$ such that $\nu(A)=0$ when $\sup A=$ $+\infty$, and $\nu(A)=-1 / 2$ otherwise, for all nonempty sets $A \subseteq \mathbb{R}$. Then $\nu$ is finitely maxitive, but not countably maxitive, and for all $f \in \mathcal{F}$,

$$
\int^{\mathrm{X}} f \mathrm{~d} \nu=\max \left\{\limsup _{x \rightarrow+\infty} f(x), \sup _{x \in \mathbb{R}} f(x)-\frac{1}{2}\right\} .
$$


In particular, $\mu: A \mapsto \int{ }^{\mathrm{x}} A_{1,0} \mathrm{~d} \nu$ on $\mathcal{P}(\mathbb{R})$ is the capacity defined in Example 1.

The next three corollaries correspond to Lemma 1, Theorem 2 (iii), and Theorem 3 (iii), respectively, since $\int^{\mathrm{x}} f \mathrm{~d} \nu=\ln \int{ }^{\mathrm{S}} e^{f} \mathrm{~d} e^{\nu}$ for all $f \in \mathcal{F}$ and all penalties $\nu$ on $\Omega$, where the logarithm and the exponential functions are extended continuously to $\overline{\mathbb{R}}_{>0}$ and $\overline{\mathbb{R}}$, respectively.

Corollary 5. Let $\nu$ be a penalty on $\Omega$, and let $\kappa$ be a cardinal. The functional $f \mapsto \int^{\mathrm{X}} f \mathrm{~d} \nu$ on $\mathcal{F}$ is $\kappa$-maxitive if and only if $\nu$ is $\kappa$-maxitive.

Corollary 6. Let $F: \mathcal{U} \rightarrow \overline{\mathbb{R}}$ be a functional such that $F(0)=0$, and define $\nu: A \mapsto F\left(A_{0,-\infty}\right)$ on $\mathcal{P}(\Omega)$. Then $F$ is finitely maxitive and additively homogeneous if and only if $\nu$ is a finitely maxitive penalty and $F: f \mapsto \int^{\mathrm{x}} f \mathrm{~d} \nu$.

Corollary 7. Let $F: \mathcal{F} \rightarrow \overline{\mathbb{R}}$ be a functional such that $F(0)=0$, and define $\nu: A \mapsto F\left(A_{0,-\infty}\right)$ on $\mathcal{P}(\Omega)$. Then $F$ is countably maxitive and additively homogeneous if and only if $\nu$ is a countably maxitive penalty and $F: f \mapsto$ $\int^{\mathrm{x}} f \mathrm{~d} \nu$.

Since $\int{ }^{\mathrm{x}} A_{0,-\infty} \mathrm{d} \nu=\nu(A)$ for all $A \subseteq \Omega$, the convex integral can be seen as an extension of the penalty $\nu$. Corollary 5 shows that this extension maintains maxitivity, while Corollaries 6-7 characterize the convex integral as maxitive, additively homogeneous functional. The value $-\infty$ plays the same role in the extension of the penalty $\nu$ by the convex integral as the value 0 does in the extension of the capacity $\mu$ by the standard, Choquet, and Shilkret integrals. The reason is that $-\infty$ is the identity element of the maximum on $\overline{\mathbb{R}}$ and the absorbing element of the addition on $\overline{\mathbb{R}} \backslash\{+\infty\}$, while 0 is the identity element of the maximum or addition on $\overline{\mathbb{R}}_{\geq 0}$ and the absorbing element of the multiplication on $\overline{\mathbb{R}}_{>0} \backslash\{+\infty\}$. By contrast, the definition of a maxitive, homogeneous integral is problematic, because the identity element of the maximum on $\overline{\mathbb{R}}$ is $-\infty$, while the absorbing element of the multiplication on $\mathbb{R}$ is 0 .

The next theorem characterizes the penalties with respect to which the convex integral is convex, and is the reason for its name.

Theorem 7. Let $\nu$ be a penalty on $\Omega$. The functional $f \mapsto \int^{\mathrm{x}} f \mathrm{~d} \nu$ on $\mathcal{F}$ is finitely convex if and only if $\nu$ is finitely maxitive.

Proof. In order to prove the "if" part, it suffices to show that if $\lambda \in(0,1)$ and $f, g \in \mathcal{F}$ are two functions such that $f+g$ and $\int \mathrm{x}_{f} \mathrm{~d} \nu+\int{ }^{\mathrm{x}} g \mathrm{~d} \nu$ are well-defined, and $x \in \mathbb{R}$, then $x+\nu(\{\omega \in \Omega: \lambda f(\omega)+(1-\lambda) g(\omega)>x\}) \leq$ $\lambda \int^{\mathrm{x}} f \mathrm{~d} \nu+(1-\lambda) \int^{\mathrm{x}} g \mathrm{~d} \nu$. Assume thus $\int^{\mathrm{x}} f \mathrm{~d} \nu+\int^{\mathrm{x}} g \mathrm{~d} \nu<+\infty$, and note that for all $\alpha \in \mathbb{R}$,

$$
\begin{aligned}
x & +\nu(\{\omega \in \Omega: \lambda f(\omega)+(1-\lambda) g(\omega)>x\}) \\
& \leq x+\nu(\{\omega \in \Omega: f(\omega)>x+(1-\lambda) \alpha\} \cup\{\omega \in \Omega: g(\omega)>x-\lambda \alpha\}) \\
& =\max \left\{\begin{array}{l}
x+\nu(\{\omega \in \Omega: f(\omega)-(1-\lambda) \alpha>x\}), \\
x+\nu(\{\omega \in \Omega: g(\omega)+\lambda \alpha>x\})
\end{array}\right\} \\
& \leq \max \left\{\int^{\mathrm{x}} f \mathrm{~d} \nu-(1-\lambda) \alpha, \int^{\mathrm{X}} g \mathrm{~d} \nu+\lambda \alpha\right\} .
\end{aligned}
$$

The desired result is obtained by letting $\alpha$ tend to $-\infty$ or $+\infty$ when $\int \mathrm{x}_{f} \mathrm{~d} \nu=$ $-\infty$ or $\int^{\mathrm{x}} g \mathrm{~d} \nu=-\infty$, respectively, and by setting $\alpha=\int^{\mathrm{x}} f \mathrm{~d} \nu-\int^{\mathrm{x}} g \mathrm{~d} \nu$ otherwise. 
The "only if" part follows from the fact that for all disjoint $A, B \subseteq \Omega$ and all $x \in \mathbb{R}_{>0}$, if $\nu(A \cup B)-x>\max \{\nu(A), \nu(B)\}$, then

$$
\begin{aligned}
\nu(A \cup B) \leq \frac{1}{2} \int^{\mathrm{X}}\left(A_{0,-\infty} \vee\right. & \left.B_{x,-\infty}\right) \mathrm{d} \nu+\frac{1}{2} \int^{\mathrm{X}}\left(A_{0,-\infty} \vee B_{-x,-\infty}\right) \mathrm{d} \nu \\
= & \frac{1}{2} \nu(A \cup B)+\frac{1}{2}(\nu(A \cup B)-x)<\nu(A \cup B) .
\end{aligned}
$$

Theorem 7 gives a necessary and sufficient condition on the penalty for the finite convexity of the integral. Countable convexity follows from finite convexity when the integral satisfies a version of Fatou's lemma, implied by monotone convergence. The next corollary corresponds to Lemma 2 (iii).

Corollary 8. Let $\nu$ be a penalty on $\Omega$. The functional $f \mapsto \iint^{\mathrm{x}} f \mathrm{~d} \nu$ on $\mathcal{F}$ satisfies monotone convergence if and only if $\nu$ is continuous from below.

Corollary 9. Let $\nu$ be a penalty on $\Omega$. The functional $f \mapsto \int \mathrm{x} f \mathrm{~d} \nu$ on $\mathcal{F}$ is countably convex if $\nu$ is countably maxitive.

Proof. Let $\left(\lambda_{n}\right)_{n \in \mathbb{N}}$ be a sequence of values $\lambda_{n} \in(0,1)$ such that $\sum_{n \in \mathbb{N}} \lambda_{n}=1$, let $\left(f_{n}\right)_{n \in \mathbb{N}}$ be a sequence of functions $f_{n} \in \mathcal{F}$ such that $\sum_{n \in \mathbb{N}} \lambda_{n} f_{n}$ and $\sum_{n \in \mathbb{N}} \lambda_{n} \int^{\mathrm{X}} f_{n} \mathrm{~d} \nu$ are well-defined, and let $\left(\mathcal{N}_{m}\right)_{m \in \mathbb{N}}$ be a nondecreasing sequence of finite sets $\mathcal{N}_{m} \subset \mathbb{N}$ such that $\bigcup_{m \in \mathbb{N}} \mathcal{N}_{m}=\mathbb{N}$. Corollary 8 and Theorem 7 imply that the functional $f \mapsto \int^{\mathrm{X}} f \mathrm{~d} \nu$ on $\mathcal{F}$ satisfies monotone convergence and is finitely convex, and thus

$$
\begin{aligned}
\int^{\mathrm{X}}\left(\sum_{n \in \mathbb{N}} \lambda_{n} f_{n}\right) \mathrm{d} \nu & =\lim _{m \rightarrow \infty} \int^{\mathrm{X}}\left(\inf _{k \in \mathbb{N}: k \geq m} \sum_{n \in \mathcal{N}_{k}} \lambda_{n} f_{n}\right) \mathrm{d} \nu \\
& \leq \liminf _{m \rightarrow \infty} \int^{\mathrm{X}}\left(\sum_{n \in \mathcal{N}_{m}} \lambda_{n} f_{n}\right) \mathrm{d} \nu \leq \sum_{n \in \mathbb{N}} \lambda_{n} \int^{\mathrm{X}} f_{n} \mathrm{~d} \nu
\end{aligned}
$$

Corollary 9 gives a sufficient condition on the penalty for the countable convexity of the integral. This condition is not necessary, as shown in the next example. A necessary and sufficient condition on the penalty for the countable convexity of the convex integral is an open problem.

Example 8. The penalty $\nu$ on $\Omega=\mathbb{R}$ defined in Example 7 is finitely maxitive, but not countably maxitive. However, the functional $f \mapsto \int^{\mathrm{X}} f \mathrm{~d} \nu$ on $\mathcal{F}$ is countably convex, as can be proved analogously to Example 4.

For positively homogeneous functionals on $\mathcal{F}^{+}$or $\mathcal{F}$, convexity and subadditivity are equivalent. But the next lemma shows that only with respect to binary penalties (i.e., penalties taking only the values $-\infty$ and 0 ) is the convex integral positively homogeneous.

Lemma 6. Let $\nu$ be a penalty on $\Omega$. The functional $f \mapsto \int^{\mathrm{x}} f \mathrm{~d} \nu$ on $\mathcal{F}$ is positively homogeneous if and only if $\nu$ is binary.

Proof. The "if" part is a direct consequence of the definitions. The "only if" part follows from the fact that for all $A \subseteq \Omega$, if $\nu(A)>-\infty$, then $\nu(A)=$ $-\int{ }^{\mathrm{X}} A_{-2 \nu(A), 0} \mathrm{~d} \nu=-2 \int \mathrm{X}_{A_{-\nu(A), 0} \mathrm{~d} \nu=0 .}$ 
Corollary 10. Let $\nu$ be a penalty on $\Omega$. The functional $f \mapsto \int^{\mathrm{x}} f \mathrm{~d} \nu$ on $\mathcal{F}$ is finitely subadditive if and only if $\nu$ is finitely maxitive and binary.

Proof. If the functional $f \mapsto \int^{\mathrm{x}} f \mathrm{~d} \nu$ on $\mathcal{F}$ is finitely subadditive, then $\nu$ is binary, since $\nu(A)=-\int \mathrm{x} A_{-2 \nu(A), 0} \mathrm{~d} \nu \geq-2 \int \mathrm{x}^{\mathrm{X}} A_{-\nu(A), 0} \mathrm{~d} \nu=0$ for all $A \subseteq \Omega$ such that $\nu(A)>-\infty$. The desired result follows now from Theorem 7 and Lemma 6.

Corollary 11. Let $\nu$ be a penalty on $\Omega$. The functional $f \mapsto \int \mathrm{x} f \mathrm{~d} \nu$ on $\mathcal{F}$ is countably subadditive if and only if $\nu$ is countably maxitive and binary.

Proof. The "if" part is implied by Corollary 9 and Lemma 6. For the "only if" part, Corollary 10 implies that $\nu$ is finitely maxitive and binary. Hence, $\nu$ is also countably maxitive when $\nu\left(\bigcup_{n \in \mathbb{N}} A_{n}\right)=-\infty$ for all sequences $\left(A_{n}\right)_{n \in \mathbb{N}}$ of pairwise disjoint sets $A_{n} \subset \Omega$ with $\nu\left(A_{n}\right)=-\infty$, and this follows from $\int^{\mathrm{X}}\left(\bigcup_{n \in \mathbb{N}} A_{n}\right)_{1,0} \mathrm{~d} \nu \leq \sum_{n \in \mathbb{N}} \int^{\mathrm{X}}\left(A_{n}\right)_{1,0} \mathrm{~d} \nu=0$.

The convex integral, interpreted as the evaluation (or expectation) of functions with respect to a penalty, satisfies a law of iterated expectations, if conditional evaluations are defined accordingly. When $F: \mathcal{F} \rightarrow \overline{\mathbb{R}}$ is a functional such that $\nu: A \mapsto F\left(A_{0,-\infty}\right)$ on $\mathcal{P}(\Omega)$ is a penalty, the conditional evaluation by $F$ of a function $f \in \mathcal{F}$ with respect to a partition $\mathcal{C} \subseteq \mathcal{P}(\Omega)$ of $\Omega$ is denoted by $F(f \mid \mathcal{C})$ and is defined as $F(f \mid \mathcal{C})=\sum_{C \in \mathcal{C}} C_{F(f \mid C), 0}$, where $F(f \mid C)=-\infty$ when $\nu(C)=-\infty$, and $F(f \mid C)=F\left(C_{f,-\infty}\right)-\nu(C)$ otherwise. When $\kappa$ is a cardinal, $F$ is said to be $\kappa$-decomposable if and only if $F(F(f \mid \mathcal{C}))=F(f)$ for all $f \in \mathcal{F}$ and all partitions $\mathcal{C} \subseteq \mathcal{P}(\Omega)$ of $\Omega$ such that $|\mathcal{C}| \leq \kappa$. The next two corollaries correspond to Lemma 3 and Theorem 5 (iii), respectively.

Corollary 12. Let $\nu$ be a penalty on $\Omega$, and let $\kappa$ be a cardinal. The functional $f \mapsto \int^{\mathrm{X}} f \mathrm{~d} \nu$ on $\mathcal{F}$ is $\kappa$-decomposable if $\nu$ is $\kappa$-maxitive.

Corollary 13. Let $\nu$ be a penalty on $\Omega$ with finite values for at least 3 pairwise disjoint subsets of $\Omega$. The functional $f \mapsto \int^{\mathrm{X}} f \mathrm{~d} \nu$ on $\mathcal{F}$ is finitely decomposable if and only if $\nu$ is finitely maxitive.

\section{References}

Artzner, P., Delbaen, F., Eber, J.-M., and Heath, D. (1999). Coherent measures of risk. Math. Finance 9, 203-228.

Ash, R. B. (1972). Real Analysis and Probability. Academic Press.

Benvenuti, P., Mesiar, R., and Vivona, D. (2002). Monotone set functions-based integrals. In Handbook of Measure Theory. North-Holland, 1329-1379.

Benvenuti, P., and Vivona, D. (2000). Comonotone aggregation operators. Rend. Mat. Appl., VII. Ser. 20, 323-336.

Bhaskara Rao, K. P. S., and Bhaskara Rao, M. (1983). Theory of Charges. A Study of Finitely Additive Measures. Academic Press.

Calvo, T., Mayor, G., and Mesiar, R., eds. (2002). Aggregation Operators. New Trends and Applications. Physica-Verlag. 
Cattaneo, M. (2007). Statistical Decisions Based Directly on the Likelihood Function. PhD thesis, ETH Zurich.

Cattaneo, M. (2013a). Likelihood decision functions. Electron. J. Stat. 7, 29242946.

Cattaneo, M. (2013b). On Maxitive Integration. Technical Report 147. Department of Statistics, LMU Munich.

Cattaneo, M. (2014). Maxitive integral of real-valued functions. In Information Processing and Management of Uncertainty in Knowledge-Based Systems, Part 1. Springer, 226-235.

Choquet, G. (1954). Theory of capacities. Ann. Inst. Fourier 5, 131-295.

Cohen, L. J. (1966). A logic for evidential support. Br. J. Philos. Sci. 17, 21-43 and $105-126$.

de Cooman, G., and Aeyels, D. (1999). Supremum preserving upper probabilities. Inf. Sci. 118, 173-212.

de Finetti, B. (1974-1975). Theory of Probability. A critical introductory treatment. 2 vols. Wiley.

Denneberg, D. (1994). Non-Additive Measure and Integral. Kluwer.

Dominiak, A. (2013). Iterated Choquet expectations: A possibility result. Econ. Lett. 120, 155-159.

Dubois, D., and Prade, H. (1988). Possibility Theory. An Approach to Computerized Processing of Uncertainty. Plenum Press.

Föllmer, H., and Schied, A. (2011). Stochastic Finance. An Introduction in Discrete Time. 3rd edn. De Gruyter.

Grabisch, M., Marichal, J.-L., Mesiar, R., and Pap, E. (2009). Aggregation Functions. Cambridge University Press.

Greco, S., and Rindone, F. (2013). Bipolar fuzzy integrals. Fuzzy Sets Syst. 220, 21-33.

Kolmogorov, A. N. (1956). Foundations of the Theory of Probability. 2nd edn. Chelsea Publishing.

Kolokoltsov, V. N., and Maslov, V. P. (1997). Idempotent Analysis and Its Applications. Springer.

Mesiar, R., Li, J., and Pap, E. (2015). Superdecomposition integrals. Fuzzy Sets Syst. 259, 3-11.

Neyman, J., and Pearson, E. S. (1928). On the use and interpretation of certain test criteria for purposes of statistical inference. Biometrika 20A, 175-240 and 263-294.

Savage, L. J. (1972). The Foundations of Statistics. 2nd edn. Dover. 
Schervish, M. J., Seidenfeld, T., and Kadane, J. B. (1984). The extent of nonconglomerability of finitely additive probabilities. Z. Wahrscheinlichkeitstheor. Verw. Geb. 66, 205-226.

Schmeidler, D. (1986). Integral representation without additivity. Proc. Am. Math. Soc. 97, 255-261.

Shackle, G. L. S. (1949). Expectation in Economics. Cambridge University Press.

Shafer, G. (1976). A Mathematical Theory of Evidence. Princeton University Press.

Shilkret, N. (1971). Maxitive measure and integration. Indag. Math. 33, 109116.

Stevens, S. S. (1946). On the theory of scales of measurement. Science 103, $677-680$

Torra, V., and Narukawa, Y. (2007). Modeling Decisions. Information Fusion and Aggregation Operators. Springer.

Wakker, P. (1989). Comonotonic Additivity for Choquet Integrals. Internal Report 89 NICI 11. Catholic University of Nijmegen.

Wang, Z., and Klir, G. J. (2009). Generalized Measure Theory. Springer.

Wu, X., and Zhou, X. (2006). A new characterization of distortion premiums via countable additivity for comonotonic risks. Insur. Math. Econ. 38, 324-334.

Yoo, K.-R. (1991). The iterative law of expectation and non-additive probability measure. Econ. Lett. 37, 145-149.

Zadeh, L. A. (1978). Fuzzy sets as a basis for a theory of possibility. Fuzzy Sets Syst. 1, 3-28. 